\title{
Polycomb subunit BMI1 determines uterine progesterone responsiveness essential for normal embryo implantation
}

\author{
Qiliang Xin, ${ }^{1}$ Shuangbo Kong, ${ }^{2,3}$ Junhao Yan, ${ }^{4}$ Jingtao Qiu, ${ }^{3}$ Bo He, ${ }^{3}$ Chan Zhou, ${ }^{3}$ Zhangli Ni, ${ }^{3}$ Haili Bao, ${ }^{3}$ Lin Huang, ${ }^{2,3}$ Jinhua Lu, ${ }^{2,3}$ \\ Guoliang Xia, ${ }^{1}$ Xicheng Liu, ${ }^{5}$ Zi-Jiang Chen, ${ }^{4}$ Chao Wang, ${ }^{1}$ and Haibin Wang ${ }^{2,3}$ \\ ${ }^{1}$ State Key Laboratory of Agrobiotechnology, College of Biological Sciences, China Agricultural University, Beijing, China. ${ }^{2}$ Reproductive Medical Center, The First Affiliated Hospital of Xiamen University, \\ Xiamen, Fujian, China. ${ }^{3}$ Fujian Provincial Key Laboratory of Reproductive Health Research, Medical College of Xiamen University, Xiamen, Fujian, China. ${ }^{4}$ Center for Reproductive Medicine, \\ Shandong Provincial Hospital Affiliated with Shandong University, Jinan, China. ${ }^{5}$ National Institute of Biological Sciences, Beijing, China.
}

\begin{abstract}
Natural and synthetic progestogens have been commonly used to prevent recurrent pregnancy loss in women with inadequate progesterone secretion or reduced progesterone sensitivity. However, the clinical efficacy of progesterone and its analogs for maintaining pregnancy is variable. Additionally, the underlying cause of impaired endometrial progesterone responsiveness during early pregnancy remains unknown. Here, we demonstrated that uterine-selective depletion of BMI1, a key component of the polycomb repressive complex-1 (PRC1), hampers uterine progesterone responsiveness and derails normal uterine receptivity, resulting in implantation failure in mice. We further uncovered genetic and biochemical evidence that BMI1 interacts with the progesterone receptor (PR) and the E3 ligase E6AP in a polycomb complex-independent manner and regulates the $\mathrm{PR}$ ubiquitination that is essential for normal progesterone responsiveness. A close association of aberrantly low endometrial BMI1 expression with restrained PR responsiveness in women who had previously had a miscarriage indicated that the role of BMI1 in endometrial PR function is conserved in mice and in humans. In addition to uncovering a potential regulatory mechanism of BMI1 that ensures normal endometrial progesterone responsiveness during early pregnancy, our findings have the potential to help clarify the underlying causes of spontaneous pregnancy loss in women.
\end{abstract}

\section{Introduction}

The implantation of the blastocyst into the maternal uterus is a crucial step in mammalian reproduction (1). Even in natural conception, the maximum chance of successful pregnancy occurring in a given menstrual cycle is limited to about $30 \%$ in humans (2) and only $50 \%-60 \%$ of all conceptions advance beyond 20 weeks of gestation (3). Among the pregnancies that are lost, implantation defects contribute to approximately $75 \%$ of these pregnancy failures (4). Blastocysts implant only when the uterus achieves a short window of receptivity $(1,5)$. These early pregnancy events are synchronized with the proliferation and differentiation of specific uterine cell types, primarily under the direction of ovarian estrogen and progesterone (P4). In mice, $\mathrm{P} 4$ priming of the uterus is obligatory for estrogen to trigger the uterus to enter into the receptive status conducive to blastocyst implantation (5). Previous molecular and genetic evidence indicates that ovarian steroid hormones via their respective nuclear receptors together with locally produced signaling molecules govern the complicated embryo-uterine crosstalk at periimplantation (1). However, the pathophysiological significance of epigenetic regulatory machinery such as histone modification during early pregnancy

Authorship note: Q. Xin, S. Kong, and J. Yan contributed equally to this work. Conflict of interest: The authors have declared that no conflict of interest exists. Submitted: January 17, 2017; Accepted: October 17, 2017. Reference information: J Clin Invest. 2018;128(1):175-189. https://doi.org/10.1172/JCI92862 has remained largely unexplored. Since the polycomb repressive complex-1-mediated (PRC1-mediated) histone H2A modification (6-8) can spatiotemporally silence the expression of homeotic transcription factors such as homeobox A10 (HOXA10) and HOXA11 (8), and given that these homeobox genes are crucial for uterine receptivity and embryo implantation in both mice and women $(9,10)$, it was conceivable that PRC1 could be a potentially important player during embryo implantation.

The mammalian PRC1 consists of several polycomb-group proteins, including 3 RING domain-containing proteins termed RING1A, RING1B, and mouse B cell-specific Mo-MLV integration site 1 (BMI1), and contains an intrinsic E3 ubiquitin ligase activity that mediates monoubiquitination of histone H2A at lysine 119, thereby resulting in PRC1-mediated gene silencing $(6-8,11)$. Biochemical analysis of these 3 RING domain-containing proteins indicates that RING1A/B is the catalytic subunit and the E3 ligase activity is formed and enhanced only when BMI1 binds to the catalytic subunit RING1A/B (7). A recent study employing pharmacological approaches demonstrated that inhibition of PRC1 activity hampers uterine decidual development in mice (12), further suggesting that PRC1-mediated histone modification is essential for early pregnancy success. However, it remained largely unknown how PRC1mediated histone modification dynamically regulates the on and off of the timing of expression of key implantation-relevant genes .

As a key component of PRC1, BMI1 was originally identified as a collaborating oncogene in the induction of lymphoma (13), and had been shown to promote tumorigenesis in numerous malig- 
nancies $(14,15)$ and stem cell self-renewal $(16,17)$. Owing to the characteristic similarity of extensive cellular proliferation and differentiation between tumorigenesis and embryo implantation, BMI1 would most likely be involved in regulating uterine function at periimplantation.

In the present investigation, combining multiple approaches, we showed herein that uterine-selective depletion of BMI1 hampers uterine $\mathrm{P} 4$ responsiveness and thus derails normal uterine receptivity, resulting in embryo implantation failure. Mechanistically, we demonstrated that BMI1 interacts with the P4 receptor (PR) and the E3 ligase E6-associated protein (E6AP) in a polycomb complex-independent manner, regulating PR ubiquitination essential for normal $\mathrm{P} 4$ responsiveness. Moreover, this essentiality of BMI1 for endometrial PR function is conserved from mouse to human, since we observed an association of aberrant BMI1 expression and low PR responsiveness in the endometrium of women who had experienced miscarriage.

\section{Results}

Uterine-selective depletion of Bmi1 results in embryo implantation failure. To address the pathophysiological significance of BMI1 during early pregnancy, we first analyzed the Bmil expression pattern in the periimplantation mouse uterus by in situ hybridization. As illustrated in Figure 1A, while Bmi1 was mainly expressed in the luminal epithelial cells on day 1 of pregnancy, its expression expanded to the uterine luminal and glandular epithelial cells and stromal cells on day 4 when the uteri enter into the receptive status. With the onset of implantation on day 5 , Bmi1 was detected in both epithelial and stromal cells surrounding the implanting blastocyst, and became more visible in the decidualizing cells on days 6-8 (Figure 1A). This dynamic uterine expression pattern of BMI1 motivated us to study its potential roles in the periimplantation events.

$B$ Bil $^{\text {loxp/loxP }}\left(\right.$ Bmil $\left.^{f / f l}\right)$ mice were crossed with PR-Cre $\left(P R^{C r e /+}\right)$ transgenic mice to achieve conditional deletion of Bmi1 (Bmi1 $\left.{ }^{d / d}\right)$ in uteri. As shown in Figure 1, B-D, Bmi1 expression can be effectively deleted in $B m i 1^{d / d}$ mice both at mRNA and protein levels. To verify the role of BMI1 in female fertility, Bmif ${ }^{f / f l}$ and Bmi1/d females were mated with fertile wild-type (WT) males, and the normal number of ovulated eggs was noted in both Bmi1 $1^{f / f l}$ and $B \mathrm{mil}^{\mathrm{d} / d}$ mice (Figure 1E). However, the litter size was markedly lower in the $\mathrm{Bmil}^{\mathrm{d} / \mathrm{d}}$ females compared with the $\mathrm{Bmi1}^{\mathrm{fl} / \mathrm{fl}}$ females (Figure $1 \mathrm{~F}$ ), suggesting that uterine BMI1 is crucial for normal female fertility. To identify the stage-specific failure of pregnancy in $\mathrm{Bmil}^{\mathrm{d} / \mathrm{d}}$ females, we subsequently analyzed the implantation status in Bmi1-mutant females, and noticed that a large portion $(>80 \%)$ of $\mathrm{Bmil}^{\mathrm{d} / \mathrm{d}}$ females exhibited implantation failure on days 5-6 of pregnancy (Figure 1, G and H). Morphologically normal blastocysts can be recovered by flushing the $\mathrm{Bmil}^{\mathrm{d} / \mathrm{d}}$ uteri without signs of attachment reaction. These results clearly indicated that uterine BMI1 is indispensable for normal embryo implantation.

BMI1 deficiency hampers uterine $P 4$ responsiveness and thus derails normal uterine receptivity. To reveal the underlying causes accounting for implantation failure upon BMI1 deficiency, we first analyzed the uterine cell proliferation versus differentiation status by BrdU incorporation assay and Ki67 immunostaining. As shown in Figure 2A, Bmi1 ${ }^{d / d}$ uterine epithelium exhibited robust aberrant proliferation accompanied with a decreased stromal proliferation on day 4 of pregnancy. We further observed an impaired epithelial membrane transformation exhibiting sustained long microvilli in $B m i 1^{d / d}$ mice (Figure 2B). This aberrant epithelial versus stromal proliferation clearly pointed to a defective uterine receptivity in the absence of BMI1.

We then analyzed the expression of uterine receptivity marker genes including those responding to estrogen (leukemia inhibitory factor [Lif], mucin $1[M u c 1]$, and lactoferrin [Ltf]) and P4 (amphiregulin [Areg], Hoxa10, and heart- and neural crest derivativesexpressed 2 [Hand2]). As illustrated in Figure 2, C-F, while Lif was normally expressed in the receptive Bmi1 ${ }^{f l / f l}$ uterine glandular epithelium on day 4, its expression was completely abolished in the absence of BMI1. In contrast, Muc1 and Ltf were abnormally induced in the uterine epithelial layer of $\mathrm{Bmil}^{d / d}$ females. Moreover, the expression of P4-target molecules such as Areg in the epithelium and Hoxa1O and Hand2 in the stroma was obviously downregulated in $\mathrm{Bmil}^{\mathrm{d} / d}$ mice.

Since Lif was not expressed in Bmil ${ }^{d / d}$ uterine glands on day 4 , we wondered whether the other glandularly expressed genes would be affected. The normal expression of Foxa2 indicated that gland identity is not disrupted in the absence of BMI1 in mice (Supplemental Figure 1; supplemental material available online with this article; https://doi.org/10.1172/JCI92862DS1). However, similar to glandular Lif expression, the expression of Prss28, Prss29, and Spink3 was obviously downregulated in day 4 $B m i 1^{d / d}$ mice, indicating an aberrant uterine receptive status in the absence of BMI1 (Supplemental Figure 1). Observations of comparable Lif expression in day 1 uteri in both $\mathrm{Bmi1}^{f / / l}$ and $B m i 1^{d / d}$ females, and absence of glandular Lif expression in nonreceptive WT uteri induced by RU486 treatment (Supplemental Figure 2, A and B) further suggested that limited Lif expression in day $4 \mathrm{Bmil}^{\mathrm{d} / \mathrm{d}}$ uterine glands is a consequence of a derailed uterine receptivity in the absence of BMI1. Moreover, supplementation of recombinant LIF protein failed to restore normal embryo implantation in $\mathrm{Bmi1}^{\mathrm{d} / \mathrm{d}}$ females (Supplemental Figure 2, C and D), suggesting that absence of glandular LIF production is not the main cause of implantation failure in the null mutant mice, which reinforced the notion that Lif may not be a direct target of BMI1 signaling.

These observations collectively indicated an exaggerated estrogen response and/or hampered $\mathrm{P} 4$ responsiveness in the $\mathrm{Bmil}^{d / d}$ uterus at periimplantation. Beyond that, these defects were not due to any alterations of circulating levels of ovarian steroid hormones, nor uterine expression status of estrogen receptor $\alpha(\mathrm{ER} \alpha)$ and PR, since we detected comparable serum levels of estradiol-17ß (E2) and P4 (Supplemental Figure 3), as well as normal uterine expression profiles of ER $\alpha$ and PR (Supplemental Figure 4) in day 4 pregnant Bmi1 ${ }^{f / f l}$ and $\mathrm{Bmil}^{d / d}$ females.

To further ascertain the participation of BMI1 in mediating estrogen versus P4 function in the uterus, we employed an ovariectomized mouse model. It was obvious that the estrogen activity in $\mathrm{Bmi1}^{\mathrm{d} / d}$ mice was comparable with that in $B m i 1^{f / / f}$ females. The expression of estrogen-regulated epithelial genes like $M u c 1$ and Ltf as well as stromal genes including insulin-like growth factor 1 (Igf1) and 11ß-hydroxysteroid dehydrogenase type 2 (Hsd11b2) was normally induced in both $\mathrm{Bmil}^{\mathrm{fl} / \mathrm{fl}}$ and $\mathrm{Bmil}^{\mathrm{d} / \mathrm{d}}$ uteri (Figure $2 \mathrm{G}$ ). In contrast, the expression levels of all tested $\mathrm{P} 4$-target genes includ- 
A

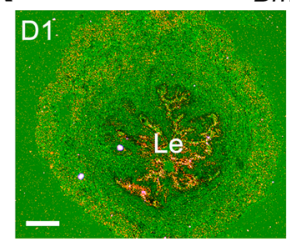

Bmi1
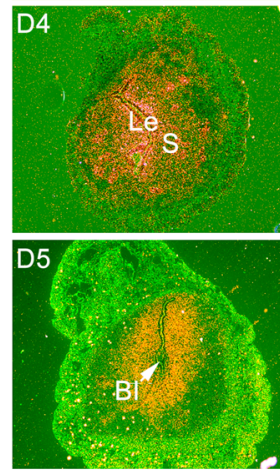

$\mathbf{E}$

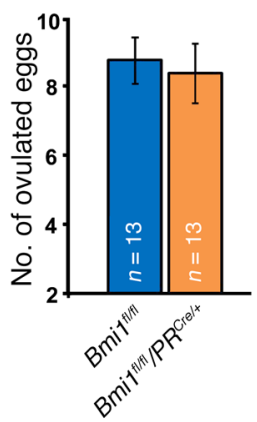

$\mathbf{F}$

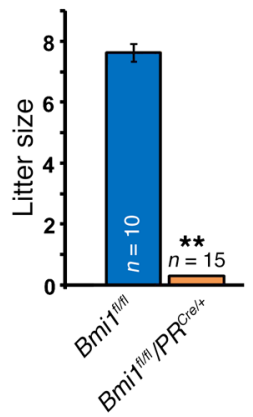

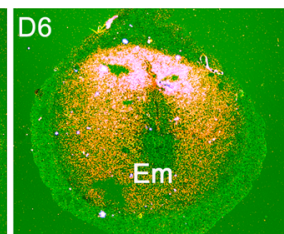
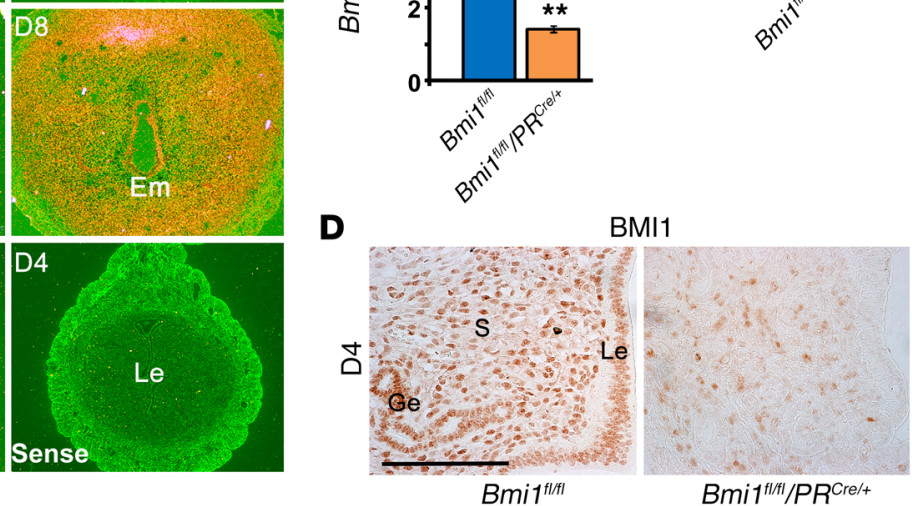

D5

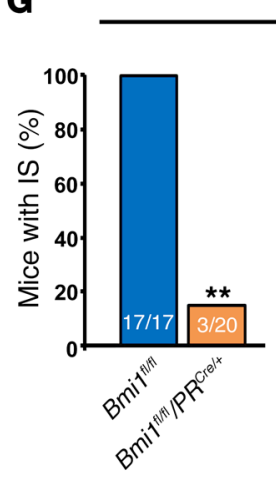

H

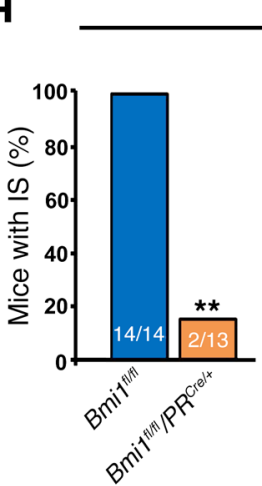

D

B

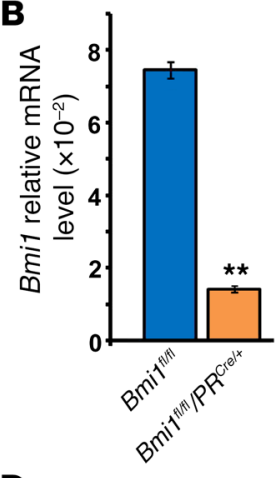

$\beta$-Acti

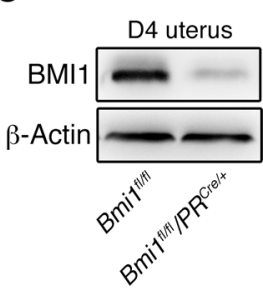

$B \mathrm{mi}^{\text {tith//PR}} / \mathrm{Prel}$

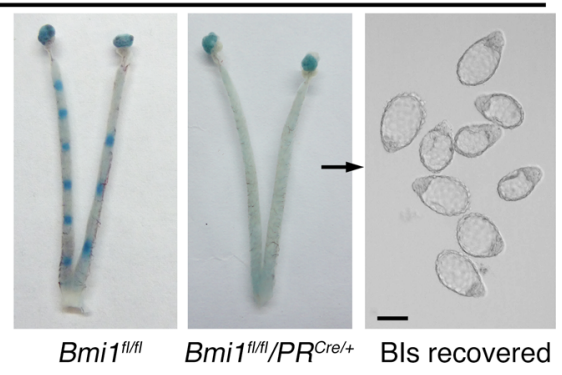

D6

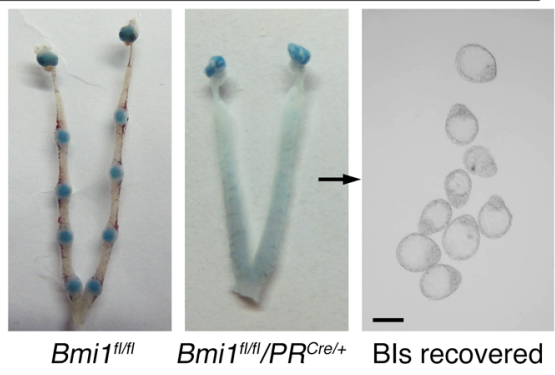

Figure 1. Uterine-selective depletion of Bmi1 results in embryo implantation failure. (A) In situ hybridization analysis reveals a spatiotemporal expression of Bmi1 in mouse uteri on days 1-8 of pregnancy. White scale bar: $100 \mu \mathrm{m}$. (B-D) Quantitative real-time PCR (B), immunoblotting (C), and immunohistochemical analysis (D) of uterine Bmi1 mRNA and protein levels in Bmi f $^{f / f l}$ and $B m i 1^{d / d}$ uteri on day 4 (D4). The values are shown as the mean $\pm \operatorname{SEM}(n=3)$. Black scale bar: 100 $\mu \mathrm{m}$. (E) Number of ovulated eggs in Bmiffl/fl and $B m i 7^{d / d}$ mice. Number within the bar indicates the number of mice tested.

(F) Average litter sizes of $B \mathrm{mi}^{\mathrm{fl} / \mathrm{fl}}$ versus $B m i 1^{d / d}$ females. Number within the bar indicates the number of mice tested. (G and $\mathbf{H}) \mathrm{A}$ large portion of $B \mathrm{mi} 7^{\mathrm{d} / \mathrm{d}}$ females exhibit implantation failure recovered with morphologically normal blastocysts upon flushing the uterine horn on days 5 (G) and $6(\mathbf{H})$ of pregnancy. IS, implantation site. Number within the bar indicates the number of mice with implantation sites per total tested mice. Data represent the mean \pm SEM. ${ }^{* *} P<0.01$, independent-samples

Student's $t$ test. Bls, blastocysts; Em, embryo; Ce, glandular epithelium; Le, luminal epithelium; S, stroma. ing Areg, Hoxa10, and Hand2 were significantly lower in P4-treated $\mathrm{Bmil}^{d / d}$ uteri (Figure $2 \mathrm{H}$ ). The $\mathrm{PR}^{\mathrm{Cr} / \mathrm{+}}$ mouse model used in this study is in fact a $P R$ heterozygote, as the Cre recombinase was knocked in downstream of the endogenous $P R$ promoter and abolished the endogenous PR expression. To exclude the possibility that hampered PR-target gene expression resulted from PR protein haploinsufficiency in $\mathrm{Bmil}^{\mathrm{d} / \mathrm{d}}$ mice, we took advantage of another Cre mouse model $\left(P R^{I R E S-C r e /+}\right)$ in which Cre was driven by the $P R$ endogenous promoter, which has an internal ribosome entry site (IRES) downstream of the $P R$ transcriptional stop codon and does not abolish the endogenous PR expression. We compared the PR protein level in these $2 \mathrm{PR}$-Cre models and the immunoblot data clearly showed a similar PR expression level in these 2 Cre models compared with the WT uterus at day 4 of pregnancy (Supplemental Figure 5A). Most importantly, Bmi1 ${ }^{f / f l} P R^{I R E S-C r e /+}$ females exhibit an implantation failure phenotype similar to that in the Bmi1 $\mathbf{1}^{f / f l}$ $P^{\text {Cre/+ }}\left(\right.$ Bmil $\left.^{d / d}\right)$ mice (Supplemental Figure 5, B and C). Therefore, we used the $\mathrm{Bmil}^{\mathrm{d} / \mathrm{d}}$ mouse model for all the following studies.

Since BMI1 was expressed in both the uterine epithelial and stromal cells, to ascertain the respective contribution of epithelial versus stromal BMI1 to P4 responsiveness, we next utilized the lactoferin-Cre $\left(\mathrm{Ltf}^{\mathrm{Cre} /+}\right)$ mouse line to establish a uterine epithelium- 
A
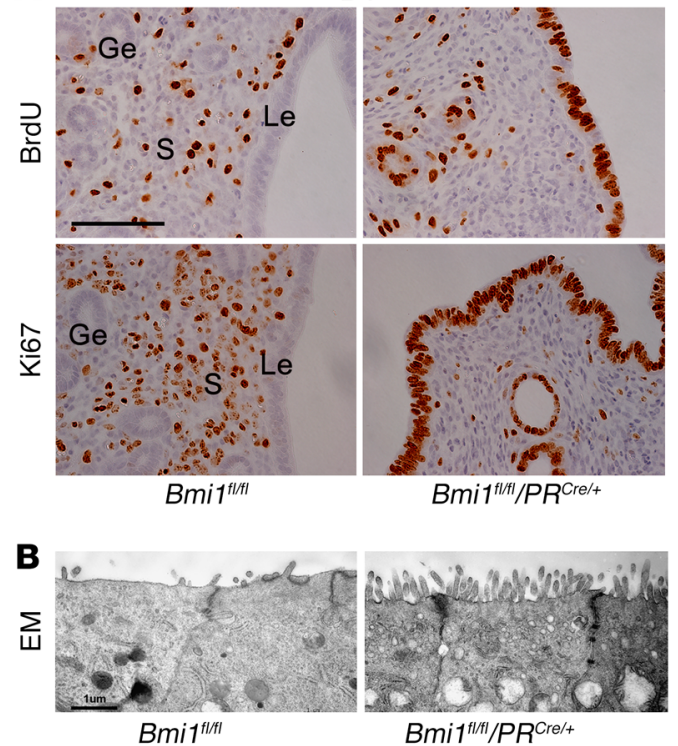

C
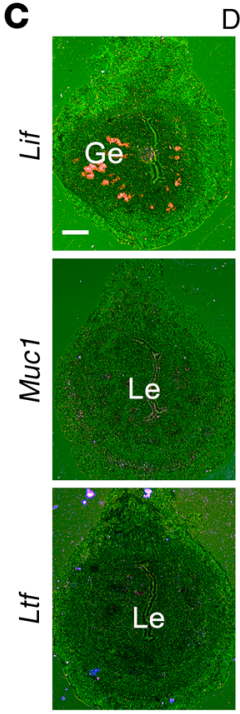

$B m i 1^{f / t h t}$
D4
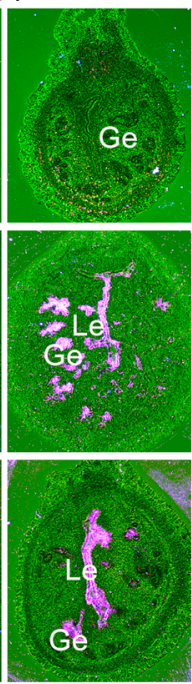

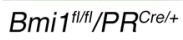
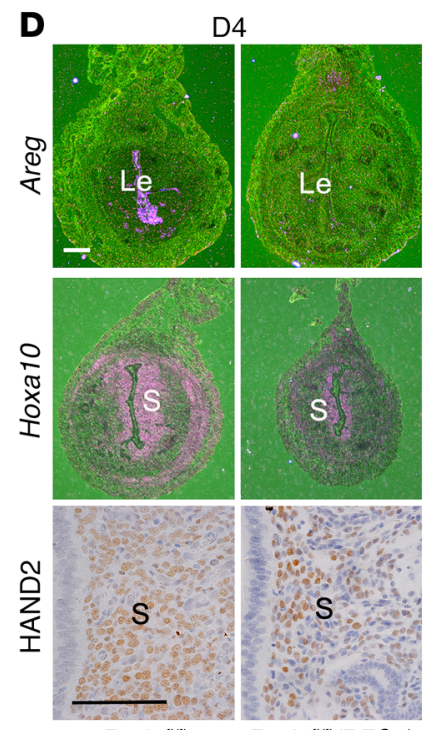

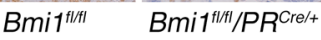
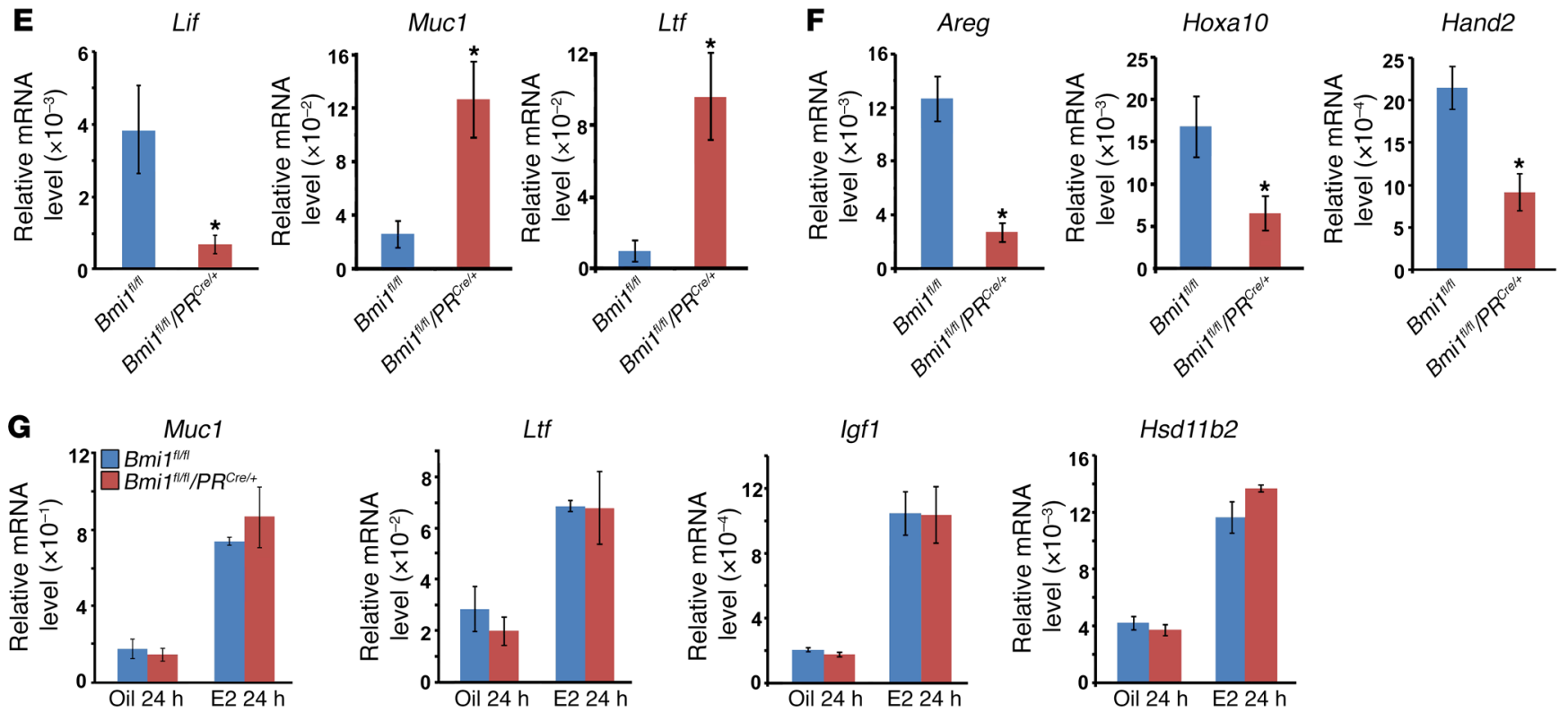

$L t f$

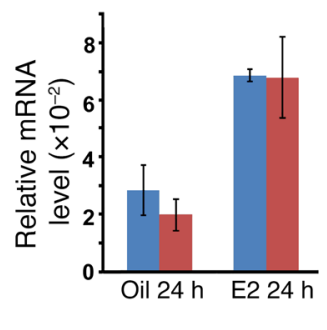

Ihh
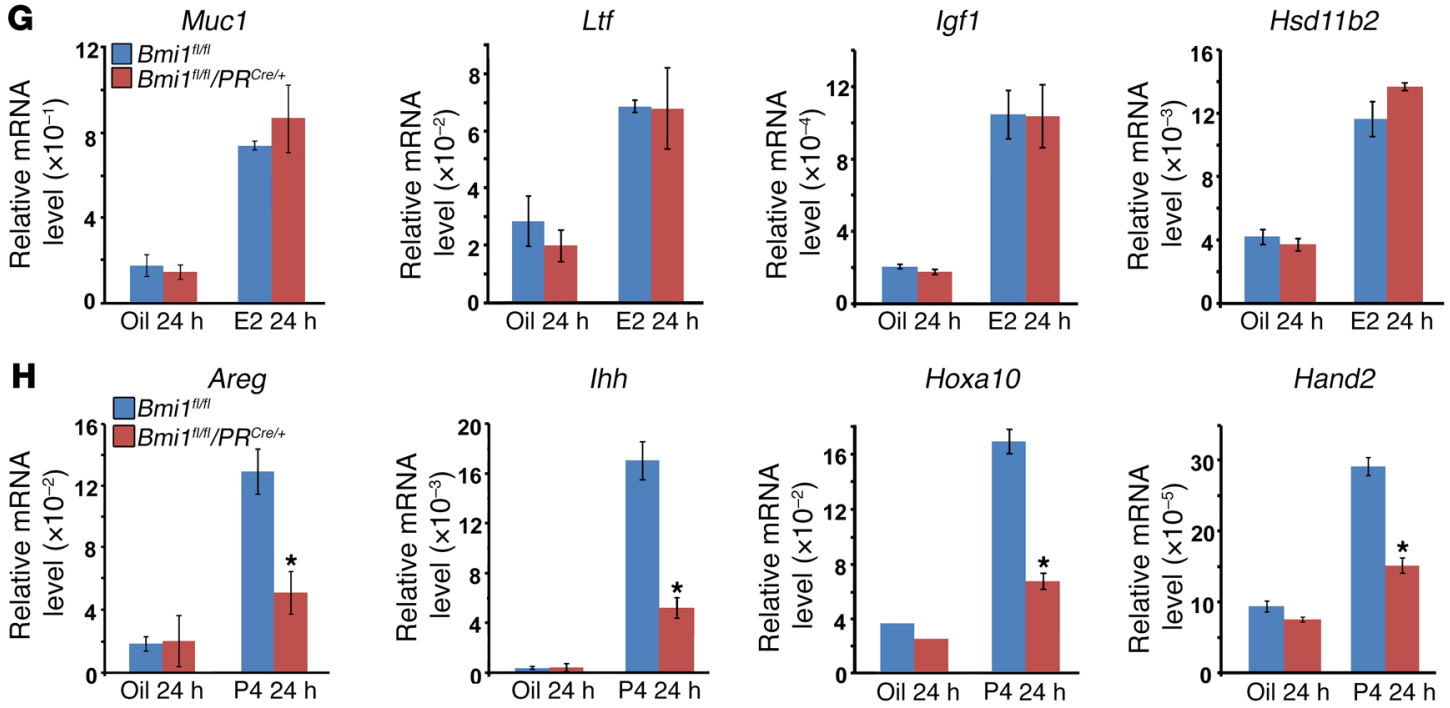

Hand2

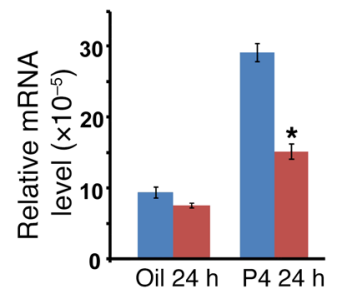

Figure 2. Bmi1 deficiency derails uterine receptivity and exhibits progesterone resistance. (A) Immunostaining analysis of BrdU and Ki67 reveals an aberrant epithelial proliferation accompanied by a decreased stromal proliferation in Bmi ${ }^{d / d}$ mouse uteri on day 4 of pregnancy. Black scale bar: $100 \mu \mathrm{m}$. (B) Electron microscopy (EM) analysis of uterine epithelial surface exhibits an impaired epithelial membrane transformation. (C-F) In situ hybridization (C and D), immunohistochemistry (D, bottom panels), and quantitative real-time PCR analysis (E and $\mathbf{F}$ ) of receptivity marker genes reveal an impaired uterine receptivity in Bmi $7^{d / d}$ females on day 4 (D4) of pregnancy. White scale bars: $100 \mu \mathrm{m}$. Black scale bar: $100 \mu \mathrm{m}$. The values are shown as the mean $\pm \mathrm{SEM}$ $(n=3)$. (G) Uterine mRNA expression of estrogen-target epithelial (Muc1, Ltff) and stromal genes (Igf1, Hsd11b2) is normally induced by E2 treatment in both $B m i f^{f / f f l}$ and $B m i 1^{d / d}$ ovariectomized mice. Data shown represent the mean \pm SEM. (H) Uterine mRNA expression of progesterone-target epithelial (Areg, I $h \mathrm{~h}$ ) and stromal genes (Hoxa10, Hand2) is largely reduced in Bmi $1^{d / d}$ ovariectomized mice in response to progesterone treatment. Data represent the mean \pm SEM $(n=3) .{ }^{*} P<0.05$, independent-samples Student's $t$ test. Ge, glandular epithelium; Le, luminal epithelium; $S$, stroma. 
A

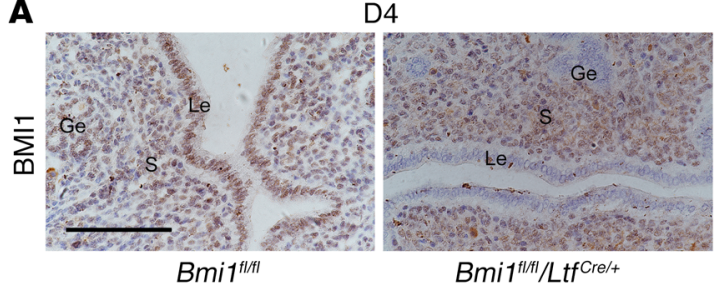

C

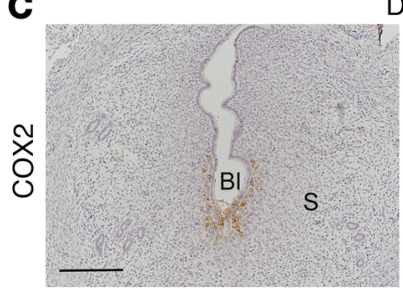

$B m i 1^{\text {thlil }}$

D
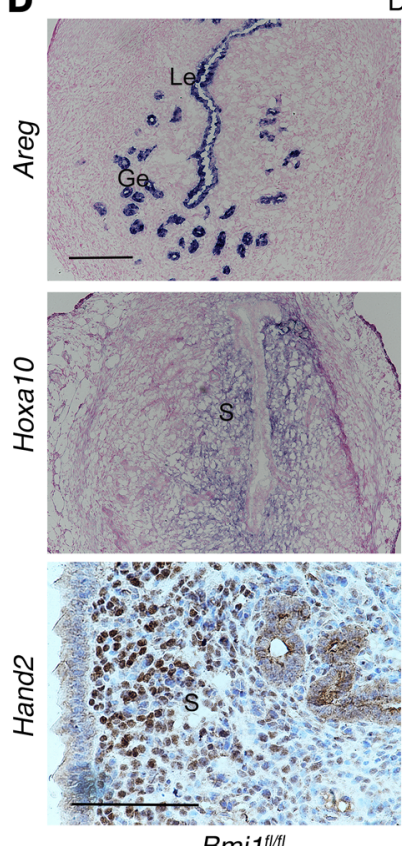

$B m i 1^{\text {th/f }}$
D5

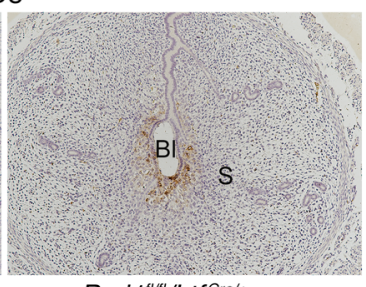

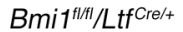

D4
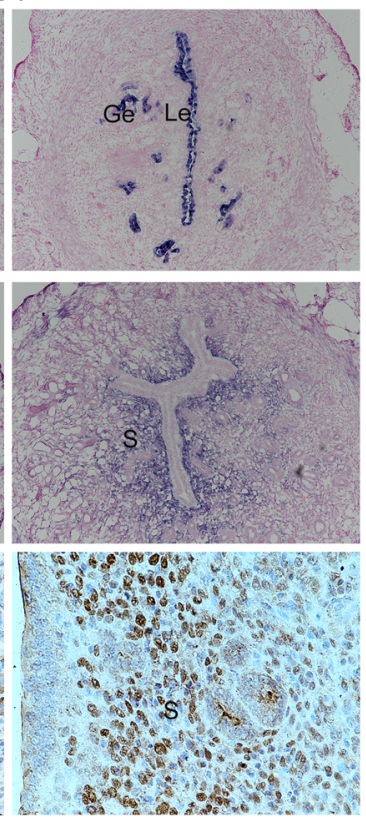

$B m i 1^{\text {fitt }} / L$ ff Crel-
B

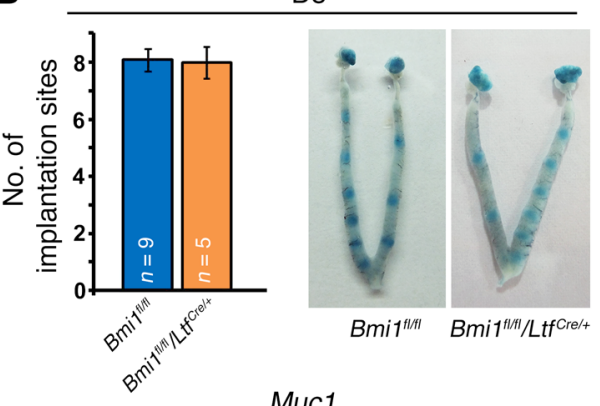

E
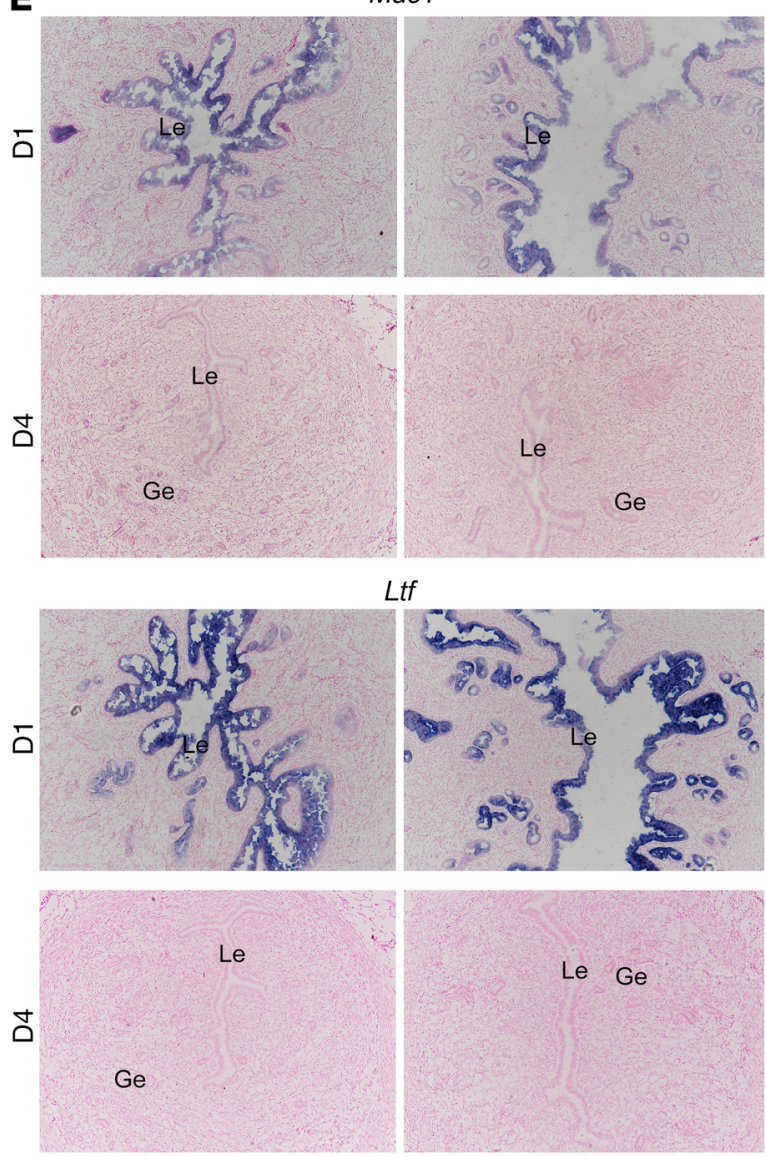

$B m i 1^{1 / n}$

\section{Le $\mathrm{Ge}$}

Figure 3. Epithelium-selective Bmi1-knockout mice exhibit normal embryo implantation and uterine expression of receptivity marker genes. (A) Immunohistochemical analysis shows the specific deletion of epithelial BMI1 in Bmi fl/fl Ltf $f^{\text {Cre/+}}$ mouse uteri. Black scale bar: $100 \mu \mathrm{m}$. (B) Number of implantation sites and representative uteri in $B m i 7^{f / f l}$ and $B m i 7^{f / f l} L t f^{c r e /+}$ mice. Number within the bar indicates the number of mice with implantation sites per total tested mice. (C) The comparable expression level of COX2 indicates normal attachment reaction in Bmif $f^{f / f l}$ and $B m i f^{f l / f l} L_{t} f^{c r e /+}$ mice. Black scale bar: $100 \mu \mathrm{m}$. ( $\mathbf{D}$ and $\mathbf{E}$ ) In situ hybridization analysis of receptivity marker genes reveal normal uterine receptivity in Bmi ${ }^{f / f l} L t f^{\text {cre/t+}}$ females on day 4 (D4) of pregnancy. Black scale bar: $100 \mu \mathrm{m}$. Bl, blastocyst; Ge, glandular epithelium; Le, luminal epithelium; S, stroma.

selective Bmi1 deletion mouse model. As shown in Figure 3A, Bmi1

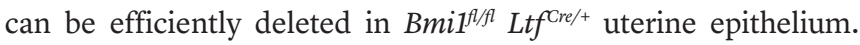
When analyzed on day 5 of pregnancy, these null females exhibited normal embryo implantation and comparable expression of COX2 (Figure 3, B and C), a marker protein for normal blastocyst attachment reaction. Meanwhile, the uterine receptivity status as determined by the related marker gene expression in both epithelium and stroma displayed no obvious differences (Figure 3, D and E). The glandularly expressed genes including Lif were also com-

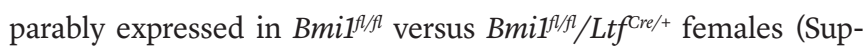
plemental Figure 6). These findings implied that epithelial BMI1 is dispensable for uterine receptivity establishment and implantation.

Based on the finding that the stromal but not epithelial BMI1 is indispensable for normal uterine $\mathrm{P} 4$ responsiveness and its deficiency induces P4 resistance and thus hampers uterine receptivity for normal embryo implantation, we subsequently asked whether or not exogenous P4 supplementation would rescue this early pregnancy loss in Bmild/d females. As shown in Supplemental Fig- 

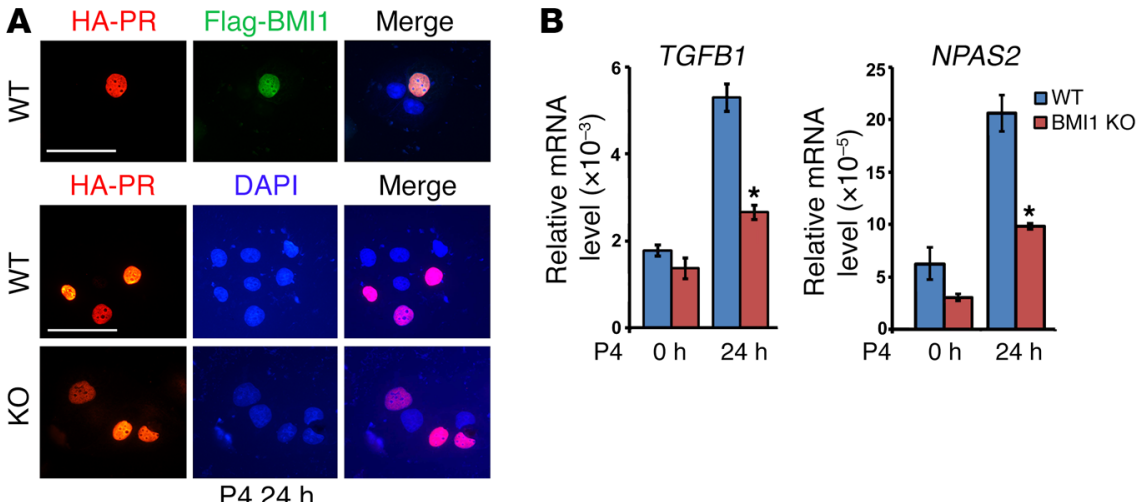

C

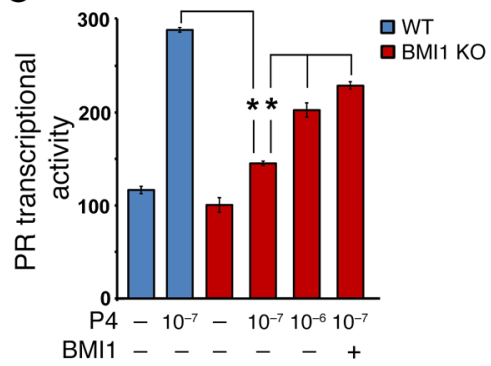

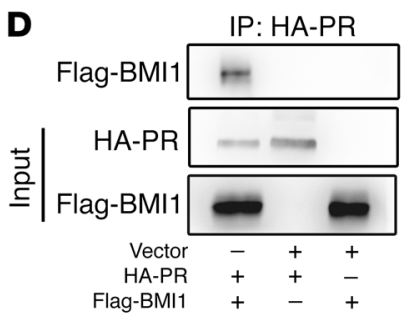

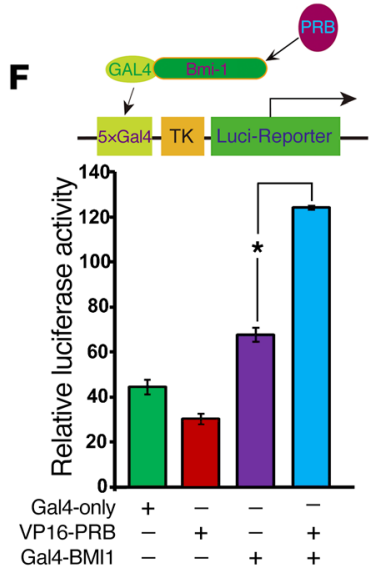

I

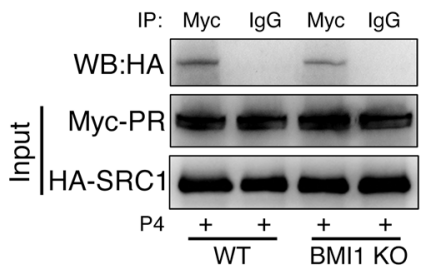

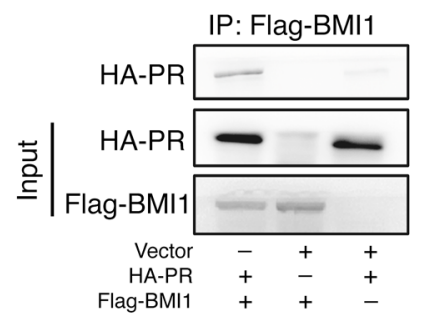

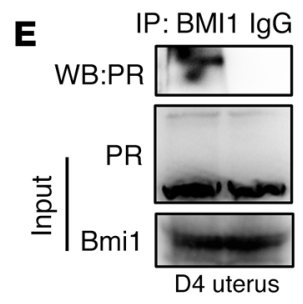

G

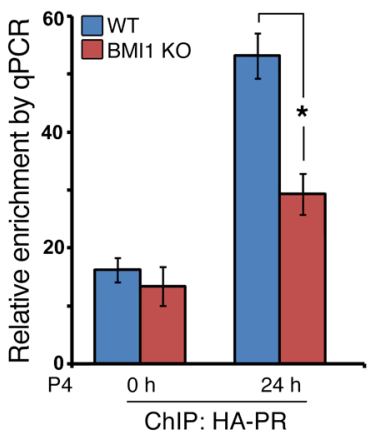

H

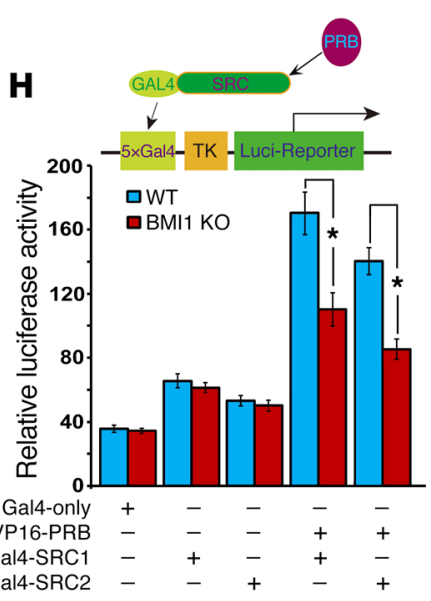

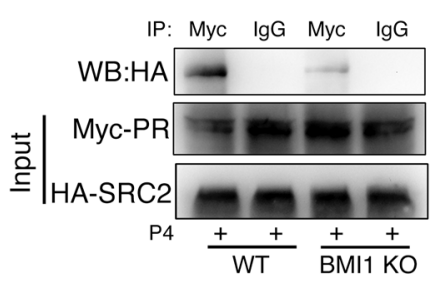

Figure 4. BMI1 facilitates PR binding to the PRE and coactivator recruitment for transcriptional activation. (A) Immunofluorescence staining of HA-PRB and Flag-BMI1 proteins in WT and BMI1-KO Ishikawa cells. Scale bars: $100 \mu \mathrm{m}$. (B) mRNA expression of progesterone-target genes TCFB1 and NPAS2 is significantly reduced in BMI1-mutant Ishikawa cells. The values are shown as the mean \pm SEM $(n=3)$. (C) The progesterone response element (PRE) luciferase reporter assay reveals a hampered PR transcriptional activity in BMI1-KO Ishikawa cells. pSV40-Renilla served as an internal control. The values are shown as the mean \pm SEM $(n=3)$. ( $D$ and $\mathbf{E})$ Coimmunoprecipitation analysis demonstrates that BMI1 can physically interact with PR in human Ishikawa cells (D) as well as in mouse receptive day 4 (D4) uteri (E). (F) Mammalian 2-hybrid assays further confirm the functional interaction of BMI1 with the PR. Vectors expressing either PRB (VP16-PRB), BMI1 (GAL4-BMI1), or GAL4-DBD only transfected into the WT Ishikawa cells. The values are shown as the mean \pm SEM $(n=3)$. (C) ChIP-qPCR analysis shows a largely reduced binding of PR to the PRE site in BMI1-KO Ishikawa cells. The values are shown as the mean $\pm \operatorname{SEM}(n=3)$. (H and I) Mammalian 2-hybrid and coimmunoprecipitation analysis reveals a significantly reduced physical association of SRC1/2 with PRB in BMI1-KO Ishikawa cells. Data shown represent the mean \pm SEM $(n=3)$. (J) Cotransfection of SRC1 or SRC2 can largely improve PR transcriptional activation in BMI1-KO Ishikawa cells. Data represent the mean $\pm \operatorname{SEM}(n=3)$. ${ }^{*} P<0.05$, independent-samples Student's $t$ test. P4, progesterone. 
A
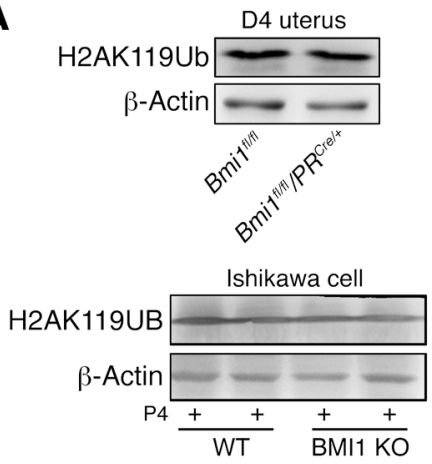

B

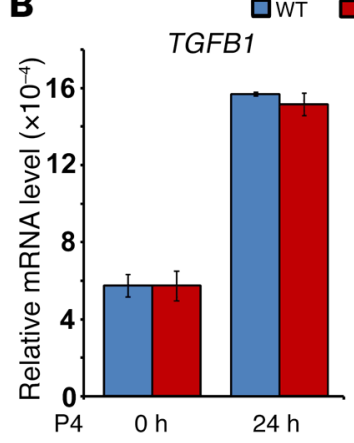

RING1A/B KO

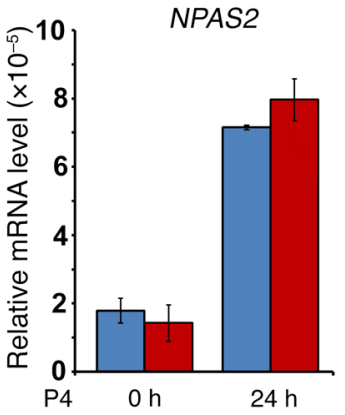

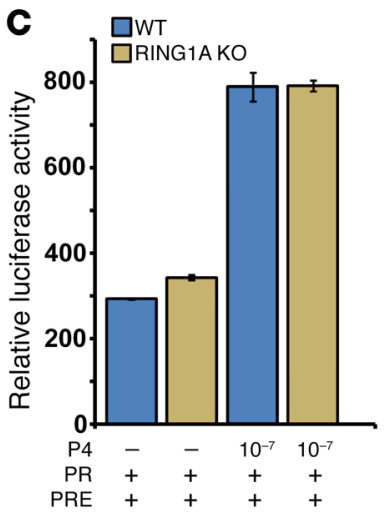
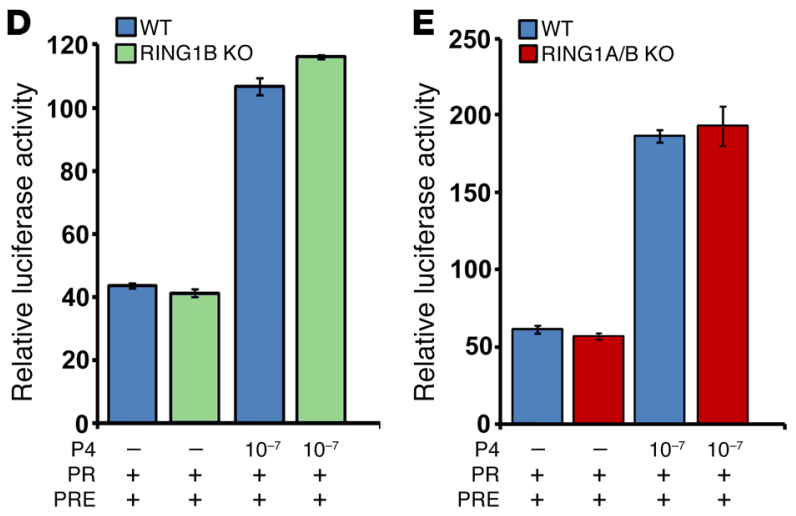

Figure 5. BMI1 regulates progesterone-PR transcriptional activity in a polycomb complex-independent manner. (A) Immunoblotting analysis shows an obviously comparable level of H2AK119 monoubiquitination in $B \mathrm{mi}^{f^{f / f l} \mathrm{l}}$ versus $B \mathrm{mi}^{\mathrm{d} / \mathrm{d}}$ mouse day 4 (D4) uteri, as well as in WT versus BMI1-KO Ishikawa cells upon progesterone treatment. $\beta$-Actin served as a loading control. (B-E) mRNA expression of progesterone-target genes TCFB1 and NPAS2 (B) as well as PR transcriptional activity accessed by PRE-luciferase reporter assay (C-E) are unaltered in RING1A-KO, RING1B-KO, and RING1A/B double-mutant Ishikawa cells. Data represent the mean $\pm \operatorname{SEM}(n=3)$. P4, progesterone. ure 7, prepriming with $\mathrm{P} 4$ for 3 consecutive days starting on day 3 could partially improve the implantation rate of Bmil-deficient females. Taken together, these findings suggested that BMI1 is necessary for optimal PR activity; its deficiency significantly hampers, but not completely abolishes PR responsiveness.

BMI1 facilitates PR binding to the PRE of the target gene promoter and coactivator recruitment for transcriptional activation. To address how BMI1 modulates PR responsiveness, we employed a human endometrial Ishikawa cell line for further mechanistic studies, since these cells can respond well to P4 treatment and show nuclear colocalization of BMI1 and PR (Figure 4A). As shown in Supplemental Figure 8, we first generated a BMI1-null mutant Ishikawa cell line utilizing the CRISPR/Cas9 knockout strategy. It is interesting to note that null mutation of BMI1 in Ishikawa cells significantly reduced the expression levels of PR target genes, such as TGFB1 and NPAS2 $(18,19)$ (Figure 4B) and hampered PR transcriptional activity as well when assessed by a $\mathrm{P} 4$ response element (PRE)-luciferase reporter assay upon P4 challenge (Figure $4 \mathrm{C})$. In line with the in vivo observation that supplementation of exogenous $\mathrm{P} 4$ can partially correct implantation defects in Bmi1/d mice, P4 exerted a dose-dependent effect triggering a subdued PR-PRE responsiveness in mutant cells (Figure 4C).

Since both BMI1 and PR exhibited nuclear colocalization in Ishikawa cells, we surmised that BMI1 may physically interact with the PR and thus ensure normal PR transcription activation. Indeed, we noted that BMI1 can physically interact with the PR both in human Ishikawa cells and mouse receptive uteri (Figure 4, D and E) when analyzed by coimmunoprecipitation (co-IP). We further conducted mammalian 2-hybrid assays to confirm the interaction of BMI1 and PR. As shown in Figure 4F, coexpression of herpes virus protein VP16/PR isoform B (VP16-PRB), but not
GAL4-BMI1, can stimulate the GAL4-BMI1-driven reporter gene expression, reinforcing the hypothesis that BMI1 is indeed able to functionally interact with the PR.

To reveal the underlying causes accounting for this obvious P4 insensitivity upon Bmi1 deficiency, we first asked whether Bmi1 deficiency would adversely affect the P4-PR binding affinity. As illustrated in Supplemental Figure 9, via ligand-receptor binding assays using radiolabeled $\left.{ }^{3} \mathrm{H}\right] \mathrm{P} 4$, we noted a normal $\mathrm{P} 4-\mathrm{PR}$ binding affinity in both $\mathrm{Bmil}^{\mathrm{d} / \mathrm{d}}$ uteri and null mutant Ishikawa cells. Moreover, although the expression of FKBP52, a cochaperone factor essential for P4-PR binding and nuclear PR signaling was significantly downregulated in the absence of Bmil, overexpression of FKBP52 failed to restore PR sensitivity upon loss of BMI1 (Supplemental Figure 10), suggesting that phenotypic defects of PR insensitivity in the absence of Bmil are irrelevant to PR cochaperone function. By contrast, we observed a significant reduction of PR binding on the PRE of target genes in the absence of BMI1 revealed by ChIP-qPCR analysis (Figure 4G). Moreover, as shown in Figure 4, $\mathrm{H}$ and I, the physical association of coactivators SRC1/2 with the PR was significantly reduced in BMI1-null cells. The results achieved via mammalian 2-hybrid assay further supported the notion that BMI1 plays a crucial role in facilitating the interaction between the PR and its coactivators SRC1/2 for normal transcriptional activation (Figure $4 \mathrm{H}$ ). In this respect, it is noteworthy that cotransfection of SRCs can partially improve PR transcriptional activity in BMI1-null cells (Figure 4J). However, the underlying mechanisms by which BMI1 regulates PR activation remain unknown.

BMI1 ensures normal $P R$ activation via modulating E6AP-mediated $P R$ ubiquitination in a polycomb complex-independent manner. Given that BMI1 is a core component of PRC1, to ascertain whether 
A

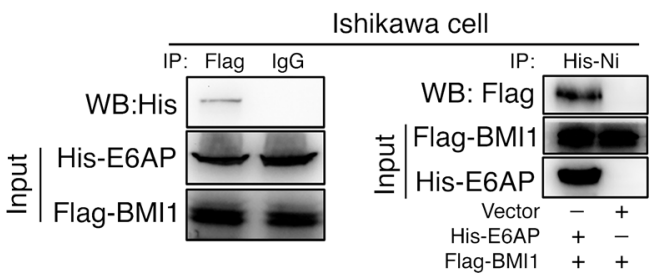

B

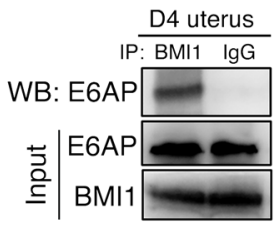

D

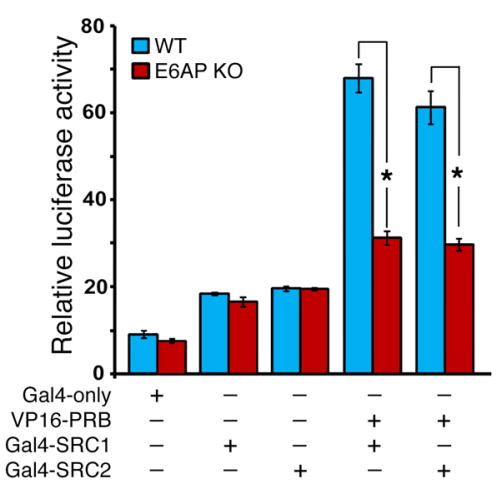

G

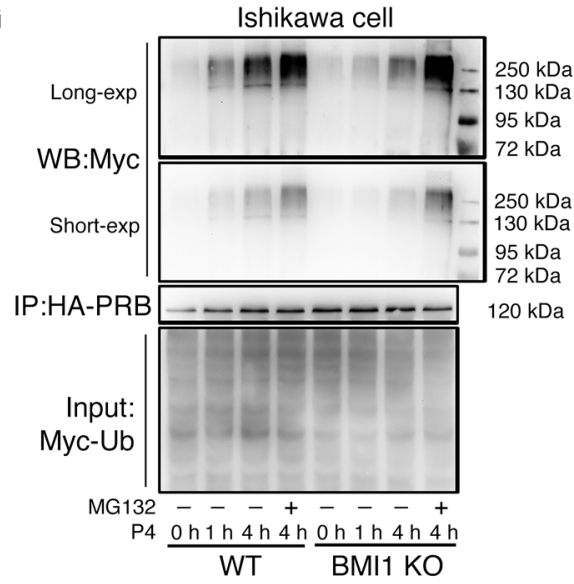

E

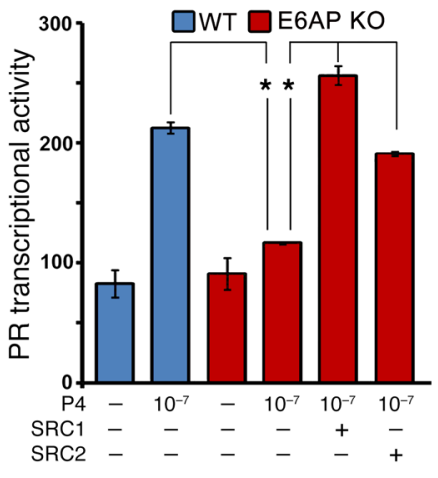

H

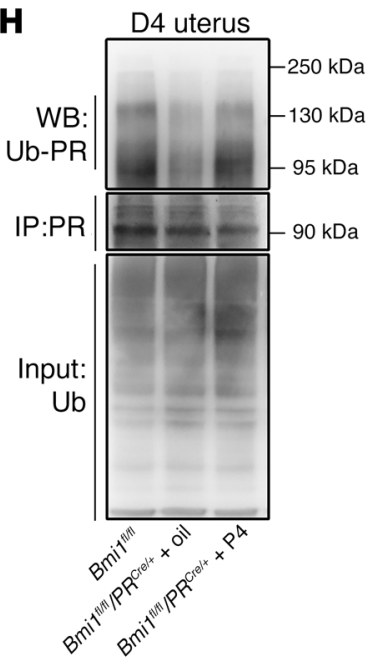

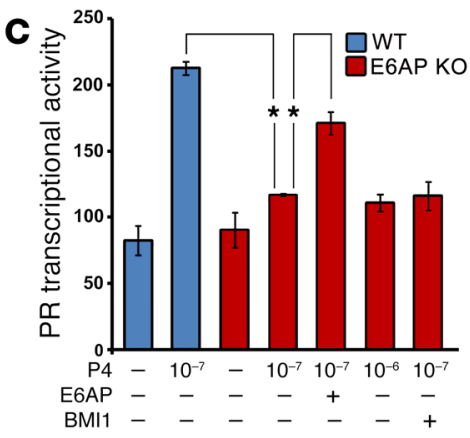

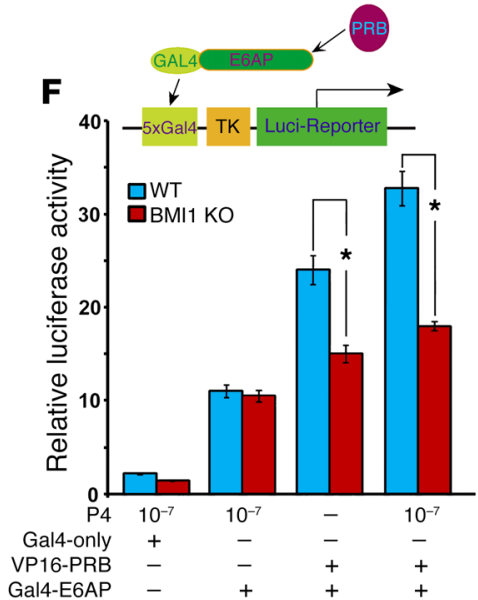

I

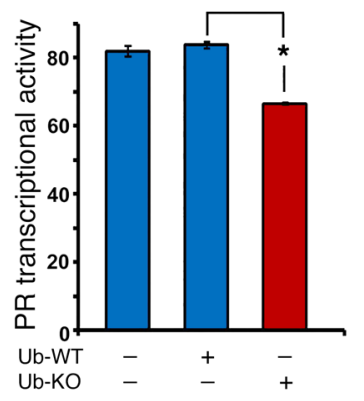

Figure 6. BMI1 ensures normal PR sensitivity via modulating E6AP-mediated PR ubiquitination. (A and B) Coimmunoprecipitation analysis reveals that BMI1 can physically interact with E6AP both in human Ishikawa cells (A) and mouse receptive day 4 (D4) uteri (B). (C) PRE-luciferase reporter assay shows a compromised PR transcriptional activity in E6AP-KO Ishikawa cells. pSV40-Renilla served as an internal control. The values are shown as the mean \pm SEM $(n=3)$. (D) Mammalian 2-hybrid analysis reveals a significantly reduced physical association of SRC1/2 with PRB in E6AP-KO Ishikawa cells. Data shown represent the mean \pm SEM $(n=3)$. (E) Cotransfection of SRC1 or SRC2 can partially improve PR transcriptional activity in E6AP-KO Ishikawa cells. The values are shown as the mean \pm SEM $(n=3)$. (F) BMI1 promotes the physical interaction of E6AP with PRB. The values are shown as the mean \pm SEM $(n=3)$. ( $(\mathbf{C}$ and $\mathbf{H})$ Ubiquitination of PRB is greatly hampered in BMl1-mutant Ishikawa cells $(\mathbf{C})$, as well as in day 4 Bmi1/d/d mouse uteri $(\mathbf{H})$. (I) PREluciferase reporter assay reveals a compromised PR transcriptional activity in WT Ishikawa cells transfected with a lysine-less Ub mutant (Ub-KO). Data represent the mean \pm SEM $(n=3) .{ }^{*} P<0.05$, independent-samples Student's $t$ test. P4, progesterone.

BMI1 regulates PR transcription activation via a PRC1-dependent manner, we first analyzed the status of PRC1-mediated H2AK119 modification in Bmi1/ ${ }^{d / d}$ uteri and knockout Ishikawa cells. To our surprise, we observed a comparable level of H2AK119 monoubiquitination in Bmi1-deficient mouse uteri and BMI1-deficient human Ishikawa cells versus those in WT mice and human endometrial cells (Figure 5A), indicating that the regulatory function of BMI1 in P4-PR nuclear signaling might be independent of PRC1 E3 ligase activity on histone H2A. To confirm this hypothesis, we generated RING1A and RING1B single-knockout, and RING1A/B 

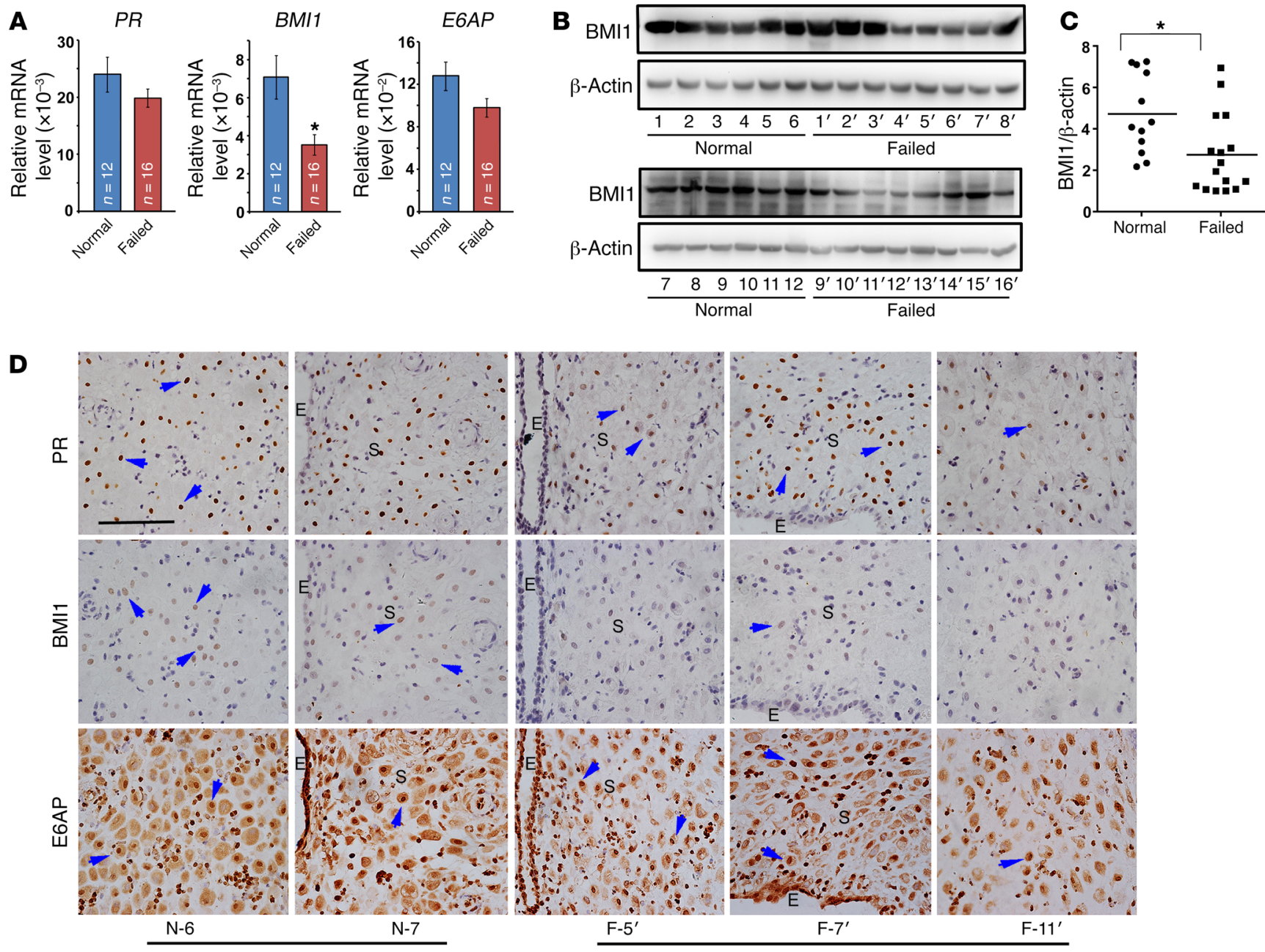

Normal

Failed
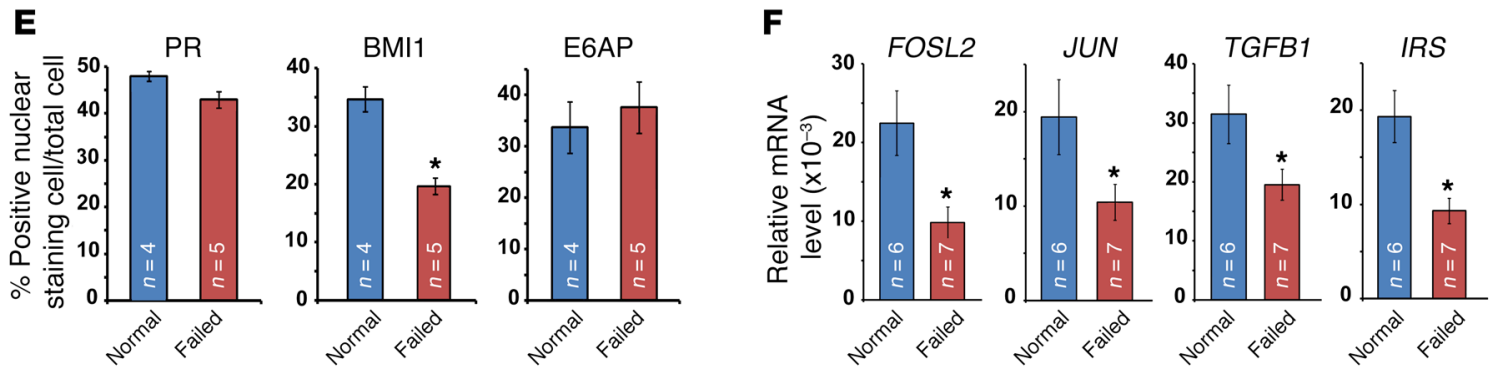

Figure 7. Aberrantly decreased endometrial BMI1 levels are often detected in spontaneously miscarrying women undergoing IVF treatment. (A)

Quantitative real-time PCR analysis of $P R, B M I 1$, and E6AP from spontaneously miscarried endometrial tissues $(n=16)$ compared with those in unwanted normal pregnancy $(n=12)$. Data shown represent the mean \pm SEM. (B and $\mathbf{C}$ ) Western blot analysis of BMl1 protein level indicates that a large portion (7 of 16) of endometrial tissues from women with spontaneous miscarriage show an obviously decreased BMI1 expression in comparison with that in normal unwanted pregnancy. In C, data are presented as the ratio of BMI1 protein level to the level of $\beta$-actin. The values are shown as the mean \pm SEM. ( $\mathbf{D}$ and E) Immunostaining analysis of endometrial PR, BMI1, and E6AP expression in women with spontaneous miscarriage versus normal unwanted pregnancy. Number within the bar indicates the number of samples tested. The values are shown as the mean \pm SEM. E, epithelium; S, stroma. Black scale bar: 100 $\mu \mathrm{m}$. (F) Endometrial mRNA expression levels of progesterone-target genes, FOSL2, JUN, TCFB1, and IRS, are significantly reduced in women with spontaneous miscarriage who exhibit lower BMI1 expression. Number within the bar indicates the number of samples tested. Data represent the mean \pm SEM $(n=3) .{ }^{*} P<0.05$, independent-samples Student's $t$ test.

double-knockout Ishikawa cell lines utilizing the CRISPR/Cas9 strategy (Supplemental Figure 11), and further observed an intact P4-PR nuclear signaling irrelevant to PRC1 functional deficiency. For example, the expression of P4-target genes TGFB1 and NPAS2 along with PR transcriptional activity assessed by PRE-luciferase reporter assay were unaltered even in RING1A/B double-mutant Ishikawa cells (Figure 5, B-E). This genetic and biochemical evidence points toward a potentially novel function of BMI1 indepen- 
A

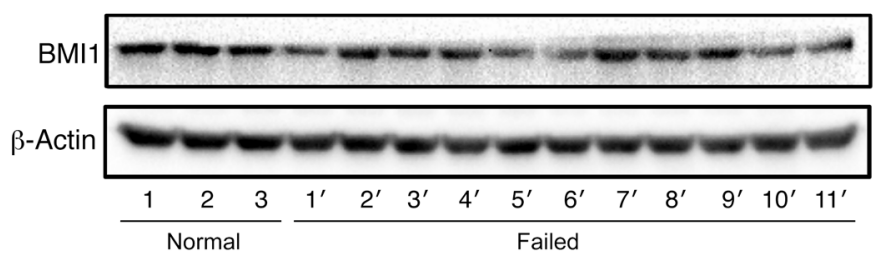

B

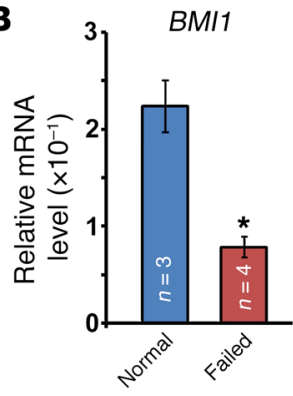

$P R$

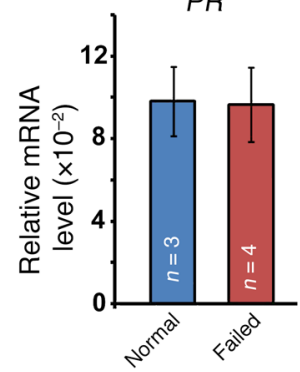

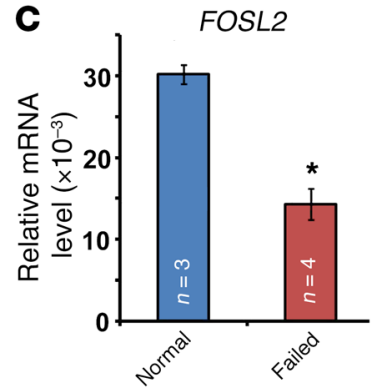
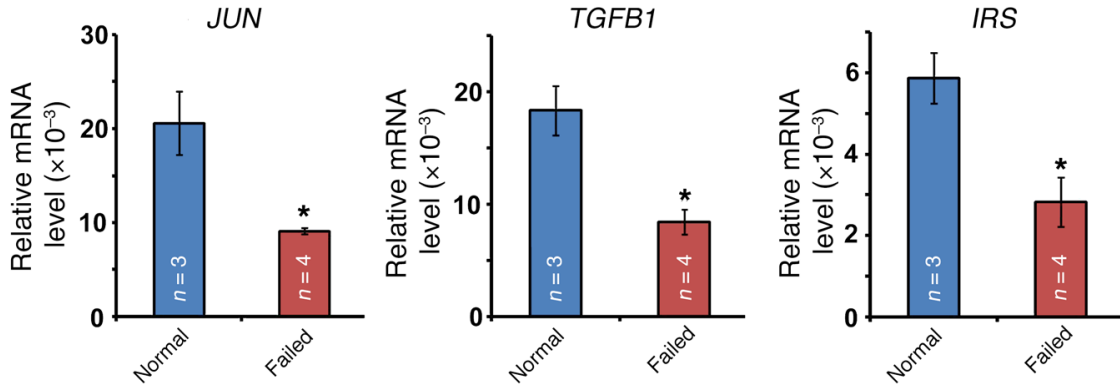

Figure 8. The decreased endometrial BMI1 level is associated with defective progesterone response in recurrent-implantation-failure patients undergoing IVF treatment. (A) Western blot analysis of BMI1 protein level indicates that a large portion (4 of 11) of endometrial tissues from women with recurrent implantation failure show an obviously decreased BMI1 expression in comparison with that in normal successful pregnancy. $\beta$-Actin served as a loading control. (B) Quantitative real-time PCR analysis of $P R$ and BMI1 from recurrent-implantation-failure patient endometrial tissues $(n=4)$ compared with those from normal successful pregnancy $(n=3)$. Data shown represent the mean \pm SEM. (C) Endometrial mRNA expression levels of progesterone-target genes, FOSL2, JUN, TGFB1, and IRS, are significantly reduced in women with recurrent implantation failure who exhibit lower BMI1 expression. Number within the bar indicates the number of samples tested. Data represent the mean \pm SEM $(n=3) .{ }^{*} P<0.05$, independent-samples Student's $t$ test.

dent of the canonical RING1A/B-PRC1 pathway with respect to PR transcriptional activity. Therefore, we next focused on searching the unknown factors bridging the gap between BMI1 and the PR.

There was early evidence that E6AP, an E3 ubiquitin ligase (20), is essential for human PR transcriptional activity (21), and can interact with ubiquitin-conjugating enzymes harboring RING domains (22). We surmised that BMI1, which contains the pivotal RING domain (7), may directly interact with E6AP, thus regulating PR modification and subsequent activation. To address this speculation, we first examined E6AP expression in mouse uteri at periimplantation. As shown in Supplemental Figure 12, E6AP was expressed in day 4 uteri at comparable levels in both $\mathrm{Bmi1}^{\mathrm{fl} / \mathrm{fl}}$ and $\mathrm{Bmi1}^{\mathrm{d} / \mathrm{d}}$ females. Co-IP analysis further revealed a physical interaction between BMI1 and E6AP in both mouse receptive uteri and human Ishikawa cells (Figure 6, A and B). Furthermore, the PR transcriptional activation indexed by PRE-luciferase reporter assay was significantly hampered in E6AP-null mutant Ishikawa cells (Figure 6C) that were generated via the CRISPR/Cas9 strategy (Supplemental Figure 13). Most importantly, while full-length E6AP restored normal PR responsiveness in E6AP-mutant cells, neither BMI1 cotransfection nor high doses of $\mathrm{P} 4$ could rescue defective PR activation (Figure 6C), suggesting that E6AP might be a downstream player of BMI1 in regulating the PR activity. Accordingly, the physical association of PR with its coactivators SRC1/2 was also significantly decreased in E6AP-null Ishikawa cells when analyzed by the mammalian 2-hybrid assay (Figure 6D). Phenotypically copying the above-mentioned observations in the absence of Bmi1, overexpression of SRC1/2 could also partially improve P4 responsiveness in E6AP-knockout cells (Figure 6E).
These findings pointed toward the same path for BMI1 and E6AP in conquering normal PR activation.

It is worth highlighting here that Bmi1 deficiency largely compromised the physical association of E6AP with PR under P4 challenge (Figure 6F). Since E6AP is an E3 ligase essential for PR function, it would therefore be interesting to ascertain the effect of Bmi1 deficiency on PR ubiquitination. As shown in Figure 6G, while the ubiquitination level of PRB was markedly induced upon P4 treatment in WT cells, the induction of PR ubiquitination was greatly hampered in BMI1-mutant cells. More excitingly, a similar reduction of PR ubiquitination was also detected in day $4 \mathrm{Bmil}^{\mathrm{d} / \mathrm{d}}$ mouse uteri, whereas an exogenous P4 supplementation that was shown to improve the implantation rate in null females can substantially restore ubiquitination of the $\mathrm{PR}$ in $\mathrm{Bmil}^{\mathrm{d} / \mathrm{d}}$ mice during early pregnancy (Figure $6 \mathrm{H}$ ). These results indicated that PR undergoing ubiquitination is conducive for its full transcription activation. This conclusion was further confirmed by our subsequent observations showing that a lysine-less Ub mutant (Ub-KO) that lacks all potential sites for polyubiquitination can significantly compromise PR transcriptional activity in WT Ishikawa cells (Figure 6I). It is conceivable that BMI1 via E6AP modulates PR polyubiquitination governing normal PR transcriptional activation. These exciting findings provoked us to explore the pathophysiological significance of BMI1 in human endometrial functions during early pregnancy.

Aberrantly decreased endometrial BMI1 expression is often detected in spontaneous miscarriage and in recurrent-implantation-failure women undergoing IVF treatments. To address whether BMI1 is involved in regulating PR responsiveness in human endometrium at periimplantation, we first analyzed endometrial expression 
With BMI1

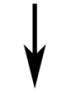

PR sensitive status

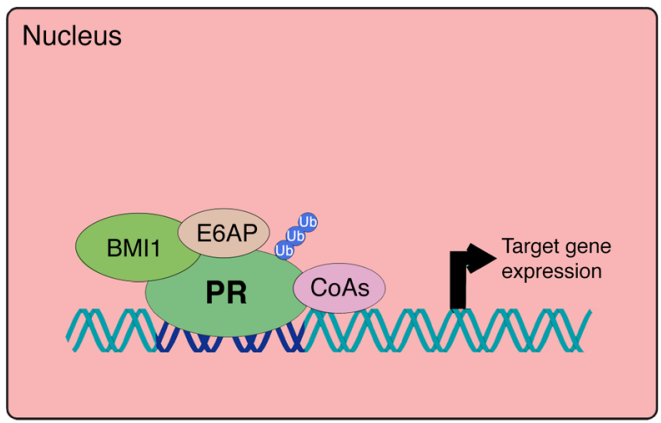

Uterine receptivity
Without BMI1

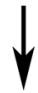

PR insensitive status

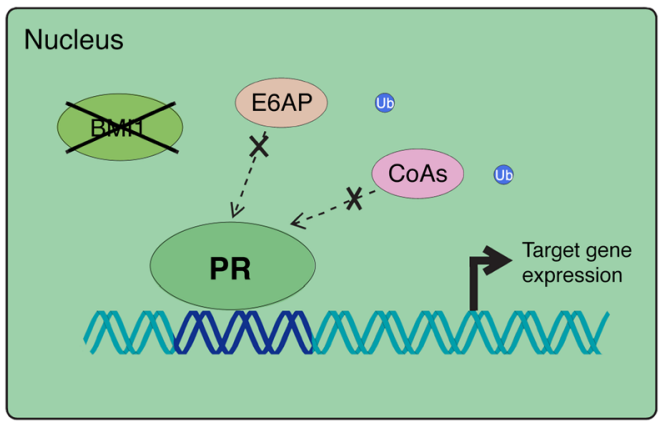

Uterine nonreceptivity
Figure 9. Illustrative working model showing how uterine BMI1 is essential for optimal PR sensitivity and thus uterine receptivity at periimplantation. BMI1 interacts with PR and E6AP in a polycomb-independent manner, regulating PR ubiquitination essential for normal progesterone responsiveness. Uterine-selective depletion of BMI1 hampers uterine progesterone responsiveness and thus derails normal uterine receptivity, resulting in implantation failure. CoAs, coactivators.

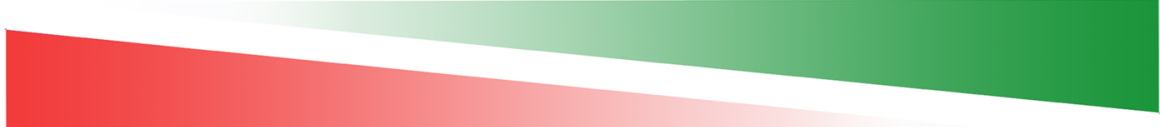

PR sensitivity

PR insensitivity

levels of Bmi1 in normal women with unwanted pregnancy (7-9 weeks; $n=12$ ) versus spontaneously miscarrying women undergoing IVF treatment (8-9 weeks; $n=16)$. As shown in Figure 7, A-C, a large portion (7 of 16) of endometrial samples from women with spontaneous miscarriage showed an obviously decreased BMI1 expression in comparison with that in normal unwanted pregnancy. Immunostaining analysis further detected a significantly reduced ratio of BMI1-positive endometrial cells in women with spontaneous miscarriage (Figure 7, D and E). By contrast, we noted comparable endometrial expression levels of PR and E6AP in both groups (Figure 7, A, D, and E). These aberrantly lower expression levels of BMI1, but not PR and E6AP, in failed pregnancy were well associated with reduced expression levels of PR-target genes including FOSL2, JUN, TGFB1, and IRS $(18,19,23)$ (Figure 7F), indicating a close correlation between BMI1 and P4-PR nuclear signaling in human endometrium during early pregnancy.

Since these patients received P4 supplementation for pregnancy maintenance during clinical therapy, which may influence overall P4-responsive genes, we further collected endometrial samples in the secretory phase (7 days after ovulation) of the normal menstrual cycle of both control participants with successful pregnancy after in vitro fertilization-embryo transfer (IVF-ET) and recurrent-implantation-failure patients. Both groups had comparable, normal P4 and E2 levels (Supplemental Table 5). It was exciting to notice that in a portion of recurrent-implantationfailure samples, the BMI1 protein levels were also obviously decreased (Figure 8A). Moreover, while BMI1 mRNA levels were significantly downregulated in these samples, $P R$ showed a comparable expression level (Figure $8 \mathrm{~B}$ ). Consistent with these data, the comprised P4-PR signaling reflected by reduced target gene mRNA levels is closely associated with decreased BMI1 expression in these endometrial samples (Figure 8C). These potentially novel findings collectively suggested that BMI1 is operative for normal
PR activity at periimplantation in both mice and humans. They also suggest that abnormal endometrial BMI1 expression with compromised PR responsiveness is at least one of the causes of spontaneous miscarriage in women.

\section{Discussion}

$\mathrm{P} 4$ is well known as a pregnancy hormone essential for preparing the uterus for embryo implantation and pregnancy maintenance (24). However, the complexity of P4-triggered PR transcriptional activation has remained largely unexplored. Employing multiple approaches, we provided herein genetic, biochemical, and pathophysiological evidence that BMI1 determines uterine P4 responsiveness via modulating $\mathrm{PR}$ ubiquitination in a polycomb complex-independent manner essential for normal embryo implantation. We demonstrated that uterine-selective depletion of Bmi1 hampers uterine $\mathrm{P} 4$ responsiveness and thus derails normal uterine receptivity, resulting in implantation failure in mice. By utilizing the Ltf-Cre mouse line to construct the epitheliumspecific Bmi1-deletion mouse model, we found that an epithelial deficiency of BMI1 did not compromise uterine P4-PR responsiveness, suggesting that stromal BMI1 is the major player contributing to normal P4 signaling for uterine receptivity and implantation at periimplantation.

Searching for the underlying mechanism, we further revealed that BMI1 interacts with PR and E6AP in a polycomb complexindependent manner, regulating $\mathrm{PR}$ ubiquitination essential for normal P4 responsiveness (Figure 9). Most importantly, this essentiality of BMI1 for endometrial PR function is conserved from mouse to human, since we observed a close association of aberrantly low BMI1 expression with retrained PR sensitivity in the endometrium of miscarrying women.

A generally accepted framework for nuclear P4 signaling is that P4 first binds with the PR by forming homodimers; dimerized PR 
complexes then translocate into the nucleus, where they bind to PREs located within the upstream promoter/enhancer sequences of target genes followed by recruitment of transcriptional coactivating factors, and eventually modulate the expression of PR-target genes $(25,26)$. Increasing evidence suggests that posttranslational modifications including phosphorylation (27) and acetylation (28) play a significant part, ultimately making the PR complex an ideal machine to regulate target gene expression. These covalent changes may affect PR stability, subcellular localization, as well as the interactions with other PR complex activators (29). In this respect, we demonstrated in the present study that BMI1 is a potentially novel regulator governing normal PR transcriptional activation. BMI1 as a critical component of the PRC1 can stimulate the complex's E3 ligase activity, which participates in the ubiquitination of lysine 119 of histone H2A to inhibit gene expression $(6,8)$. However, we surprisingly found that BMI1 deficiency exerted apparently no effects on the overall levels of H2AK119 monoubiquitination in both mouse uteri and human endometrial cells. Increasing evidence suggests that BMI1 can function in a polycomb complexindependent manner in various processes $(30,31)$. Therefore, it is conceivable that BMI1 may function in a noncanonical manner independent of PRC1 during early pregnancy. This notion is consistent with the observation that a functional silencing of PRC1 upon genetic ablation of both RING1A and RING1B did not interfere with normal PR transcriptional activity. By contrast, independent of polycomb function, BMI1 regulates PR ubiquitination for normal transcriptional activation. This posttranslational modification of the PR is not ascribed to a direct enzymatic activity of BMI1, since BMI1 displays no detectable ubiquitin ligase activity alone (6). A more likely scenario is that BMI1 mediates the physical association of E6AP with PR, further regulating PR ubiquitination for transcriptional activation. In fact, BMI1 is essential for normal functional interaction of the PR with E6AP. A previous study has shown that E6AP is involved in human PRB ubiquitination for its timely turnover via the proteasome pathway during mammary gland development (32). In this regard, we observed a comparable expression level of PR proteins upon the loss of Bmi1, pointing toward a disassociation of PR ubiquitination by the BMI1-PR-E6AP complex from the process of PR protein turnover. This is consistent with previous reports showing that posttranslational protein modifications by ubiquitin proteins are vital for functional activation of modified proteins, independent of proteasome-mediated degradation mechanisms (33). Collectively, our findings with respect to BMI1 add a potentially new regulatory layer contributing to the complexity and preciseness of PR transcriptional activation.

Although natural and synthetic progestogens have been extensively used in pregnant patients or patients undergoing infertility treatments for various indications such as prevention of unexplained recurrent pregnancy loss and threatened abortion (34, 35), it remained controversial whether P4 supplementation would increase the chance of successful implantation and ongoing pregnancy among women with a history of unexplained recurrent miscarriages $(35,36)$. Debate is still ongoing with regard to the best administration timing (luteal phase or first trimester of pregnancy) and method of $\mathrm{P} 4$ supplementation for better implantation rate and term pregnancy outcomes in these patients (36). It is conceivable that aberrant expression and/or function of PR transcriptional cofactors such as BMI1 could be a potential cause for implantation failure and unexplained spontaneous miscarriage even under regular P4 supplementation during IVF. Therefore, our current findings raise the important notion that any disturbance of the PR transcriptional activation machinery would hamper $\mathrm{P} 4$ responsiveness regardless of normal levels of circulating P4 and endometrial PR proteins, eventually inducing early pregnancy loss.

Nonetheless, here we provided genetic, biochemical, and pathophysiological evidence that BMI1 is an essential player in optimizing PR sensitivity during early pregnancy. Besides uncovering a potentially novel regulatory mechanism ascribed to BMI1 in governing endometrial P4 responsiveness at periimplantation, our findings have high clinical relevance, since aberrantly low BMI1 expression is well associated with largely reduced PR responsiveness in the endometrium of women who experience miscarriage. A better understanding of the regulatory network of the BMI1-PR-E6AP complex may help to develop related targeted therapy for clinical treatment of miscarriage in women.

\section{Methods}

Animals and treatments. Bmi $1^{f l / l}$ mice, provided by Rongwen Xi (National Institute of Biological Sciences, Beijing, China), were generated by introducing 2 loxP sites into introns flanking exons 2-8. A detailed description of this mouse line will be published elsewhere. $P R^{\text {Cre/+}}(37)$, Ltf $^{\text {Cre/+}}(38)$, and $P R^{I R E S-C r e /+}$ (39) mouse models were utilized to delete $B m i 1$ in the uteri. The $P^{\text {IRES-Cre/+ }}$ mouse model was purchased from The Jackson Laboratory (stock number 017915). Uterine-specific knockout mice were generated by crossing Bmi1 $1^{f / f l}$ with different Cre mouse models in the C57/129 background. All mice were housed in the Animal Care Facility of Xiamen University, in accordance with the guidelines for the care and use of laboratory animals. Female mice at least 8 weeks old were mated with fertile WT males to induce pregnancy (vaginal plug = day 1 of pregnancy). The number of implantation sites, demarcated by distinct blue bands, was recorded. Mice that failed to recover any embryos were excluded from the statistical analysis. Mouse blood samples were collected on day 4 in the morning and serum P4 as well as E2 levels were measured by radioimmunoassay (40). For P4 treatment, each mouse was subcutaneously injected with $2 \mathrm{mg}$ in $100 \mu \mathrm{l}$ of sesame oil at 9:00 am for 3 consecutive days starting on day 3 until the pregnant mice were sacrificed for analysis (41). The mice were treated with RU486 (50 $\mu \mathrm{g} /$ mouse) on day 3 , and sacrificed on day 4 for detecting Lif expression. To determine potential alterations of estrogen- and P4-target gene expression, mice were ovariectomized irrespective of the estrous cycle. After resting for 10 days, mice were given a subcutaneous injection of E2 (100 ng/mouse) or P4 (2 mg/mouse) dissolved in sesame oil. LIF protein was provided by Ziqiang Li (Beijing VDJBio Co. LTD). To determine the efficiency of recombinant LIF protein in inducing implantation, delayed-implanting mice were treated with a subdose of estrogen (1.5 ng/mouse) combined with the LIF protein, and compared with the lowest single dose of estrogen ( $3 \mathrm{ng} / \mathrm{mouse}$ ) to induce implantation (42). Bmi1 ${ }^{f / f l} \mathrm{PR}^{\mathrm{Cre} /+}$ mice received intraperitoneal injection of LIF protein ( $50 \mu \mathrm{g} / \mathrm{mouse}$ ) once per day from day 4 until sacrifice on day 6 to check the implantation status.

In situ hybridization. In situ hybridization was performed as previously described (43). Mouse-specific cRNA probes labeled with isotope or digoxin for Bmi1, Areg, Muc1, Ltf, Lif, Spink3, Foxa2, Prss28, 
Prss29, and Hoxa1O were used for hybridization. Cryosections hybridized with sense probes served as negative controls.

Immunostaining. Immunohistochemistry was performed in $5-\mu \mathrm{m}-$ thick, $10 \%$ neutral buffered formalin-fixed paraffin-embedded sec-

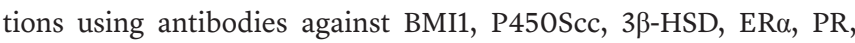
HAND2, Ki67, BrdU, and E6AP. A Histostain-SP Kit (Zhongshan Golden Bridge Biotechnology) was applied to visualize the antigen. For immunofluorescence staining, $4 \%$ formaldehyde-fixed Ishikawa cells were incubated with anti-BMI1, -HA, and -flag antibodies. Specific secondary antibodies were utilized to detect the antigen and DAPI was applied to identify cell nuclei (44). The images were captured by using a Leica DM2500 light microscope. Antibodies used are listed in Supplemental Table 1.

Western blot analysis. Protein extraction and Western blot analysis were performed as described previously (44). Antibodies against BMI1, RING1A, RING1B, E6AP, H2AK119Ub, ER $\alpha$, PR, flag, HA, FKBP52, $\beta$-actin, Myc, ubiquitin, and His were used. $\beta$-Actin served as a loading control. See complete unedited blots in the supplemental material. Antibodies used are listed in Supplemental Table 1.

Cell culture. The Ishikawa cells were maintained at $37^{\circ} \mathrm{C}$ in an atmosphere of $5 \% \mathrm{CO}_{2} / 95 \%$ air in DMEM-F12 medium supplemented with $10 \%$ (v/v) FBS. Lipofectamine 3000 (Invitrogen) was used for plasmid transfection experiments in Ishikawa cells based on the manufacturer's instructions.

Quantitative real-time PCR. Quantitative real-time PCR was performed as described previously (40). Total RNA was extracted from uterine tissues or cells using TRIzol reagent (Invitrogen) following the manufacturer's protocol. A total of 3-5 $\mu$ g RNA was used to synthesize cDNA. Quantitative real-time PCR was performed with SYBR Green (Takara) on an ABI PRISM 7500 system. All expression values were normalized against Gapdh. All PCR primers are listed in Supplemental Table 2.

Co-IP binding assays. Co-IP assays were performed as previously described (40). Anti-HA mouse mAb (agarose conjugated) $(20 \mu \mathrm{l}$, Abmart, m20013), anti-flag M2 Affinity Gel (20 $\mu$ l, MilliporeSigma, A2220), anti-BMI1, anti-PR, anti-Myc, and His-Select Nickel Affinity Gel (25 $\mu$ l, MilliporeSigma, P6611) were used. Whole-cell protein (0.5 to $2 \mathrm{mg}$ ) was used for each co-IP. After overnight incubation at $4^{\circ} \mathrm{C}$ with 3-5 $\mu$ g antibody, Protein A/G Agarose beads (Thermo Fisher Scientific, 20422) were incubated $2-3$ hours at $4^{\circ} \mathrm{C}$ and then were washed with IP washing buffer 3-5 times. Immunoprecipitated proteins were separated by SDS-PAGE and analyzed by Western blot, using antibodies against Flag, HA, PR, c-Myc, and BMI1. The control immunoprecipitation was performed by incubating the lysates with respective IgG (Zhongshan Golden Bridge Biotechnology).

Vector construction, transfection, and luciferase assay. The human full-length cDNA for BMI1 was inserted into P-CMV-3XFlag vector for the production of Flag fusion protein. Full-length cDNAs for SRC1/2, FKBP52, and E6AP were inserted into P-CMV-HA or P-CMV-Myc-His vector for the production of $\mathrm{HA}$ and $\mathrm{Myc}-\mathrm{His}$ fusion proteins, respectively. PRB expression vectors as well as ubiquitin-related expression vectors were provided by Xuemin Zhang (National Center of Biomedical Analysis, Beijing, China). The reporter plasmid PRE-Tk-Luc was provided by Weinian Shou (Indiana University, Indianapolis, Indiana, USA).

Gal4-BMI1, Gal4-SRC1/2, Gal4-E6AP, VP16-PR, VP16-E6AP, and the Gal4 upstream activation sequence (Gal4-UAS) reporter constructs or a control vector (containing the Gal4 DNA-binding domain [Gal4-DBD] only) were transfected into Ishikawa cells as previously described (45). Gal4 luciferase reporter activity and PRE-Tk-Luc were measured. Ishikawa cells were cultured in 24-well plates and then transfected with a total of $1.2 \mu$ g plasmid. All constructs were transiently transfected into Ishikawa cells using Lipofectamine 3000. Total cell lysates were prepared 24 hours after P4 treatment and luciferase activity was measured using the Dual-Luciferase Reporter Assay System (Promega). Firefly luciferase activity was normalized with respect to Renilla luciferase activity. All samples were analyzed in triplicate.

Genomic deletions of BMI1, E6AP, RING1A, RING1B, and RING1A/B genes via the CRISPR/Cas9 system in human Ishikawa endometrial cells. Genomic deletions of the respective genes in Ishikawa cells employing the CRISPR/Cas9 system were performed as previously described (46). The Cas9 and sgRNA plasmid pX458 were obtained from Addgene (plasmid number 48138). The guide RNAs (gRNAs) (Supplemental Table 3) targeting human BMI1, RING1A, RING1B, RING1A/B, and E6AP were designed to knock out these genes using the CRISPR Design Tool (sgRNAs were synthesized, annealed, and ligated to the pX458 plasmid that was digested with BbsI [New England Biolabs]). The insert gRNA sequences were verified by Sanger sequencing of the plasmids. After the transfection of target plasmid into the Ishikawa cells, FACS-isolated single $\mathrm{GFP}^{+}$cells were plated into the 96-well plate. After the single cells were propagated to a sufficient population size, the knockout efficiency was identified by DNA sequencing of genomic DNA and Western blot detection of target protein.

$P R$ ligand binding assay. Lysates from the WT and BMI1-deficient uterine tissue as well as Ishikawa cells were prepared as described above (47). The lysates were used for the binding assay without freezing. Both saturation and competition assays were performed using $\left[{ }^{3} \mathrm{H}\right]$ P4. In each case, binding was allowed to occur for 20 hours on ice. Nonspecific binding was determined in the presence of excess unlabeled hormone. Protein-bound radioactivity was isolated using 1\% dextrancoated charcoal in $10 \mathrm{mM}$ HEPES buffer ( $\mathrm{pH}$ 7.4). Specific binding was normalized to protein content. Unbound ligand was removed by incubation with dextran-coated charcoal, and bound radioactivity in the supernatant was measured by liquid scintillation counting.

Transmission electron microscopy. Tissues were cut into 1- to 3-mm pieces, and then were fixed in 3\% glutaraldehyde and $2.0 \%$ formaldehyde in cacodylate buffer with $2 \mathrm{mM} \mathrm{CaCl}_{2}$, washed, postfixed in $1 \%$ osmium tetroxide $\left(\mathrm{OsO}_{4}\right)$, and embedded in EMbed812 (Electron Microscopy Sciences) for ultrastructural analysis under a Hitachi H-7600 transmission electron microscope (Hitachi High-Technologies America, Inc.)(40).

$P R$ ubiquitination assay. In vitro and in vivo ubiquitination assays were performed as previously described (48). For in vitro ubiquitination assay, HA-PRB and Myc-Ub were cotransfected into WT or knockout Ishikawa cells with Lipofectamine 3000. Then, the cells were treated with P4 (100 nM) for 0-4 hours, and subsequently harvested using IP RIPA lysis buffer. The MG-132 (Sigma M8699, $20 \mu \mathrm{M}$ ) was added into the cultured cells 1 hour before the collection. PRB proteins were immunoprecipitated with an anti-HA antibody, the proteins were released from the beads by boiling in SDS-PAGE sample buffer, and PRB ubiquitination was analyzed by immunoblotting with anti-Myc antibody. For in vivo ubiquitination assay, PR proteins from mouse uteri were immunoprecipitated with an anti-PR antibody (Cell Signalling Technology), the proteins were released from the beads by boiling in SDS-PAGE sample buffer, and PR ubiquitination was analyzed by immunoblotting with anti-Ub antibody. 
Endometrial sample collection. Female patients, who were diagnosed as infertile and sought IVF treatment at the Center for Reproductive Medicine, Shandong Provincial Hospital Affiliated with Shandong University, were recruited and gave their written informed consent. The study was approved by the Ethics Committee Review Board of Reproductive Medicine, Shandong University. For collection of human endometrial tissues in the women who had experienced miscarriage, the tissues were collected when the participants were under surgery to term the pregnancy. Normal control endometrial biopsies were collected from the decidua of 12 women, which had fetal heart activity on ultrasound scan (USS) at 7-9 weeks. Sixteen decidual samples were collected from spontaneously miscarrying women without fetal heart activity on USS 8-9 weeks after IVF. Embryonic villi chromosome analysis (EVCA) was performed to ensure a normal embryonic genetic background. A detailed description of patient clinical parameters is listed in Supplemental Table 4. For the collection of human endometrial tissue during the menstrual cycle, the control group included participants who had achieved successful pregnancy after IVF-ET practices, and only patients who had undergone at least 3 IVF-ET failures, in which no less than 10 high-quality 8-cell embryos or 5 blastocysts were transferred in total, were included for recurrent-implantation-failure group. All patients in these 2 groups had a good basal hormonal level and a good response to hormonal stimulation (more than 8 oocytes/ oocyte retrieval). Participants were instructed not to use hormonal therapy for at least for 3 months. Endometrial biopsies were collected using a Pipelle de Cornier device on day 7 after ovulation (follicle development was evaluated by B-mode ultrasound to determine the ovulation day). A detailed description of patient clinical parameters is provided in Supplemental Table 5. Each tissue sample was divided into 2 pieces and immediately frozen in liquid nitrogen or fixed in $4 \%$ formalin for immunohistochemical analyses. All tissues were separated from trophoblasts without prior pharmaceutical induction.

Statistics. Statistical analysis was performed with SPSS 11.5. Comparison of means is presented by making use of the independentsamples Student's $t$ test. The data are shown as the mean \pm SEM. P values less than 0.05 were considered statistically significant.

Study approval. All animal studies were performed with the approval of the Xiamen University Animal Care and Use Program. Ethics approval for the collection and use of human endometrial samples was given by the Ethics Committee Review Board of Reproductive Medicine, Shandong University.

\section{Author contributions}

QX, SK, ZJC, CW, and HW were responsible for experimental design and data interpretation. QX, JQ, BH, CZ, ZN, HB, LH, and JL performed experiments. JY and XL contributed reagents and data interpretation. QX, SK, GX, and HW prepared the manuscript.

\section{Acknowledgments}

We are grateful to Francesco DeMayo (National Institute of Environmental Health Sciences, Research Triangle Park, North Carolina, USA), Sudhansu K. Dey (Cincinnati Children's Hospital Medical Center, Cincinnati, Ohio, USA), and Rongwen Xi (National Institute of Biological Sciences, Beijing, China) for providing us with the $\mathrm{PR}^{\mathrm{Cr} /+}, \mathrm{Ltf}^{\mathrm{Cre} /+}$, and $\mathrm{Bmi1}^{\mathrm{fl} / \mathrm{fl}}$ mice, respectively. We would like to thank Xuemin Zhang (National Center of Biomedical Analysis, Beijing, China) for providing the PRB and ubiquitin-related expression vectors. We are also grateful to Weinian Shou (Indiana University) and Qing Zhang (Nanjing University, Nanjing, China) for providing us with the PRE-TkLuc reporter vector and Flag-Ub vector, respectively. We thank Xiangzhi Li (Shandong University, Jinan, China) for proofreading the manuscript. This work was supported by the National Key R\&D Program of China (grant 2017YFC1001402 to H.W.; 2017YF0104603 to S.K. and J.L.) and the National Natural Science Foundation (grants 81330017 and 81490744 to H.W., 81601285 to S.K., and 31600945 to J.L.).

Address correspondence to: Haibin Wang, Chengyi building of Xiamen University Medical College, Xiang'an South Road, Xiang'an District, Xiamen 361102, China. Phone: 86.592.2880501; Email: haibin.wang@vip.163.com. Or to: Chao Wang, Center for Life Sciences, College of Biological Sciences, China Agricultural University, No. 2 Yuanmingyuan West Road, Haidian District, Beijing 100193, China. Phone: 86.10.62733435; Email: wangcam@126.com. Or to: Zi-Jiang Chen, Center for Reproductive Medicine, Shandong Provincial Hospital Affiliated to Shandong University, No.157 Jingliu Road, Jinan 250001, China. Phone: 86.531.85651190; Email: chenzijiang@hotmail.com. Or to: Xicheng Liu, National Institute of Biological Sciences, 7 Science Park Road ZGC Life Science Park, Beijing 102206, China. Phone: 86.10.80726688; Email: liuxicheng@nibs.ac.cn.
1. Dey SK, et al. Molecular cues to implantation. Endocr Rev. 2004;25(3):341-373.

2. Zinaman MJ, Clegg ED, Brown CC, O'Connor J, Selevan SG. Estimates of human fertility and pregnancy loss. Fertil Steril. 1996;65(3):503-509.

3. Norwitz ER, Schust DJ, Fisher SJ. Implantation and the survival of early pregnancy. $N$ Engl J Med. 2001;345(19):1400-1408.

4. Wilcox AJ, et al. Incidence of early loss of pregnancy. N Engl J Med. 1988;319(4):189-194.

5. Wang H, Dey SK. Roadmap to embryo implantation: clues from mouse models. Nat Rev Genet. 2006;7(3):185-199.

6. Wang H, et al. Role of histone H2A ubiquitination in polycomb silencing. Nature. 2004;431(7010):873-878.

7. Li Z, Cao R, Wang M, Myers MP, Zhang Y, Xu
RM. Structure of a Bmi-1-Ring1B polycomb group ubiquitin ligase complex. J Biol Chem. 2006;281(29):20643-20649.

8. Cao R, Tsukada Y, Zhang Y. Role of Bmi-1 and Ring1A in H2A ubiquitylation and Hox gene silencing. Mol Cell. 2005;20(6):845-854.

9. Taylor HS, Arici A, Olive D, Igarashi P. HOXA10 is expressed in response to sex steroids at the time of implantation in the human endometrium. J Clin Invest. 1998;101(7):1379-1384.

10. Gendron RL, Paradis H, Hsieh-Li HM, Lee DW, Potter SS, Markoff E. Abnormal uterine stromal and glandular function associated with maternal reproductive defects in Hoxa-11 null mice. Biol Reprod.1997;56(5):1097-1105.

11. Buchwald G, van der Stoop P, Weichenrieder O, Perrakis A, van Lohuizen M, Sixma TK. Structure and E3-ligase activity of the Ring-Ring complex of polycomb proteins Bmi1 and Ring1b. EMBO J. 2006;25(11):2465-2474.

12. Bian F, Gao F, Kartashov AV, Jegga AG, Barski A, Das SK. Polycomb repressive complex 1 controls uterine decidualization. Sci Rep. 2016;6:26061.

13. van Lohuizen M, Verbeek S, Scheijen B, Wientjens E, van der Gulden H, Berns A. Identification of cooperating oncogenes in $\mathrm{E}$ mu-myc transgenic mice by provirus tagging. Cell. 1991;65(5):737-752.

14. Bruggeman SW, et al. Bmi1 controls tumor development in an Ink4a/Arf-independent manner in a mouse model for glioma. Cancer Cell. 2007;12(4):328-341.

15. Leung $\mathrm{C}$, et al. Bmil is essential for cerebellar development and is overexpressed in human medulloblastomas. Nature. 
2004;428(6980):337-341.

16. Lessard J, Sauvageau G. Bmi-1 determines the proliferative capacity of normal and leukaemic stem cells. Nature. 2003;423(6937):255-260.

17. Park IK, et al. Bmi-1 is required for maintenance of adult self-renewing haematopoietic stem cells. Nature. 2003;423(6937):302-305.

18. Rubel CA, Lanz RB, Kommagani R, Franco HL, Lydon JP, DeMayo FJ. Research resource: Genome-wide profiling of progesterone receptor binding in the mouse uterus. Mol Endocrinol. 2012;26(8):1428-1442.

19. Tamm-Rosenstein K, Simm J, Suhorutshenko M, Salumets A, Metsis M. Changes in the transcriptome of the human endometrial Ishikawa cancer cell line induced by estrogen, progesterone, tamoxifen, and mifepristone (RU486) as detected by RNA-sequencing. PLoS ONE. 2013;8(7):e68907.

20. Scheffner M, Huibregtse JM, Vierstra RD, Howley PM. The HPV-16 E6 and E6-AP complex functions as a ubiquitin-protein ligase in the ubiquitination of p53. Cell. 1993;75(3):495-505.

21. Nawaz Z, et al. The Angelman syndromeassociated protein, E6-AP, is a coactivator for the nuclear hormone receptor superfamily. Mol Cell Biol.1999;19(2):1182-1189.

22. Zheng N, Wang P, Jeffrey PD, Pavletich NP. Structure of a c-Cbl-UbcH7 complex: RING domain function in ubiquitin-protein ligases. Cell. 2000;102(4):533-539.

23. Kaya HS, Hantak AM, Stubbs LJ, Taylor RN, Bagchi IC, Bagchi MK. Roles of progesterone receptor A and $B$ isoforms during human endometrial decidualization. Mol Endocrinol. 2015;29(6):882-895.

24. Cha J, Sun X, Dey SK. Mechanisms of implantation: strategies for successful pregnancy. Nat Med. 2012;18(12):1754-1767.

25. Pratt WB, Toft DO. Steroid receptor interactions with heat shock protein and immunophilin chaperones. Endocr Rev. 1997;18(3):306-360.

26. Rosenfeld MG, Lunyak VV, Glass CK. Sensors and signals: a coactivator/corepressor/epi- genetic code for integrating signal-dependent programs of transcriptional response. Genes Dev. 2006;20(11):1405-1428.

27. Denner LA, Weigel NL, Maxwell BL, Schrader WT, O'Malley BW. Regulation of progesterone receptor-mediated transcription by phosphorylation. Science. 1990;250(4988):1740-1743.

28. Chung HH, Sze SK, Tay AS, Lin VC. Acetylation at lysine 183 of progesterone receptor by $\mathrm{p} 300$ accelerates DNA binding kinetics and transactivation of direct target genes. J Biol Chem. 2014;289(4):2180-2194.

29. Abdel-Hafiz HA, Horwitz KB. Post-translational modifications of the progesterone receptors. J Steroid Biochem Mol Biol. 2014;140:80-89.

30. Lavial F, et al. Bmi1 facilitates primitive endoderm formation by stabilizing Gata6 during early mouse development. Genes Dev. 2012;26(13):1445-1458.

31. Wei F, et al. BMI1 attenuates etoposide-induced G2/M checkpoints via reducing ATM activation. Oncogene. 2015;34(23):3063-3075.

32. Ramamoorthy S, Dhananjayan SC, Demayo FJ, Nawaz Z. Isoform-specific degradation of PR-B by E6-AP is critical for normal mammary gland development. Mol Endocrinol.2010;24(11):2099-2113.

33. Wang $\mathrm{Q}$, et al. The E3 ubiquitin ligase AMFR and INSIG1 bridge the activation of TBK1 kinase by modifying the adaptor STING. Immunity. 2014;41(6):919-933.

34. El-Zibdeh MY, Yousef LT. Dydrogesterone support in threatened miscarriage. Maturitas. 2009;65(Suppl 1):S43-S46.

35. Coomarasamy A, Williams H, Rai R. Progesterone in women with recurrent miscarriages. NEnglJMed. 2016;374(9):894.

36. Coomarasamy A, et al. A randomized trial of progesterone in women with recurrent miscarriages. N Engl JMed. 2015;373(22):2141-2148.

37. Soyal SM, et al. Cre-mediated recombination in cell lineages that express the progesterone receptor. Genesis. 2005;41(2):58-66.
38. Daikoku T, Ogawa Y, Terakawa J, Ogawa A, DeFalco T, Dey SK. Lactoferrin-iCre: a new mouse line to study uterine epithelial gene function. Endocrinology. 2014;155(7):2718-2724.

39. Yang CF, et al. Sexually dimorphic neurons in the ventromedial hypothalamus govern mating in both sexes and aggression in males. Cell. 2013;153(4):896-909.

40. Zhang S, et al. Uterine Rbpj is required for embryonic-uterine orientation and decidual remodeling via Notch pathway-independent and -dependent mechanisms. Cell Res. 2014;24(8):925-942.

41. Tranguch S, Wang H, Daikoku T, Xie H, Smith DF, Dey SK. FKBP52 deficiency-conferred uterine progesterone resistance is genetic background and pregnancy stage specific. JClin Invest. 2007;117(7):1824-1834.

42. Ma WG, Song H, Das SK, Paria BC, Dey SK. Estrogen is a critical determinant that specifies the duration of the window of uterine receptivity for implantation. Proc Natl Acad Sci U S A. 2003;100(5):2963-2968.

43. Wang H, et al. Fatty acid amide hydrolase deficiency limits early pregnancy events. J Clin Invest. 2006;116(8):2122-2131.

44. Lu J, et al. A positive feedback loop involving Gcm1 and Fzd5 directs chorionic branching morphogenesis in the placenta. PLoS Biol. 2013;11(4):e1001536.

45. Hu H, et al. CRL4B catalyzes H2AK119 monoubiquitination and coordinates with PRC2 to promote tumorigenesis. Cancer Cell. 2012;22(6):781-795.

46. Ran FA, Hsu PD, Wright J, Agarwala V, Scott DA, Zhang F. Genome engineering using the CRISPRCas9 system. Nat Protoc. 2013;8(11):2281-2308.

47. Tranguch S, et al. Cochaperone immunophilin FKBP52 is critical to uterine receptivity for embryo implantation. Proc Natl Acad Sci U S A. 2005;102(40):14326-14331.

48. Yuan L, et al. Deubiquitylase OTUD3 regulates PTEN stability and suppresses tumorigenesis. Nat Cell Biol. 2015;17(9):1169-1181. 